\title{
GENERAL RELATIVISTIC RADIATIVE TRANSFER IN HOT ASTROPHYSICAL PLASMAS: \\ A CHARACTERISTIC APPROACH
}

\author{
Silvia Zane ${ }^{1}$, Roberto Turolla ${ }^{2}$, Luciano Nobili ${ }^{2}$ and Myris Erna ${ }^{2}$ \\ ${ }^{1}$ International School for Advanced Studies, Trieste \\ Via Beirut 2-4, 34014 Miramare-Trieste, Italy \\ ${ }^{2}$ Department of Physics, University of Padova \\ Via Marzolo 8, 35131 Padova, Italy
}




\begin{abstract}
In this paper we present a characteristic method for solving the transfer equation in differentially moving media in a curved spacetime. The method is completely general, but its capabilities are exploited at best in presence of symmetries, when the existence of conserved quantities allows to derive analytical expressions for the photon trajectories in phase space. In spherically-symmetric, stationary configurations the solution of the transfer problem is reduced to the integration of a single ordinary differential equation along the bi-parametric family of characteristic rays. Accurate expressions for the radiative processes relevant to continuum transfer in a hot astrophysical plasma have been used in evaluating the source term, including relativistic e- $\mathrm{p}$, e-e bremsstrahlung and Compton scattering. A numerical code for the solution of the transfer problem in moving media in a Schwarzschild spacetime has been developed and tested. Some applications, concerning "hot" and "cold" accretion onto non-rotating black holes as well as static atmospheres around neutron stars, are presented and discussed.
\end{abstract}

Subject headings: accretion, accretion disks - numerical methods - radiative transfer - relativity 


\section{INTRODUCTION}

Radiative transfer in high energy, fast moving plasmas in a strong gravitational field is today at the basis of a large number of currently interesting astrophysical applications; accretion onto compact objects, jets, stellar collapse and supernova expanding envelopes are just some examples of this.

Since the pioneering works by Thomas (1930), Simon (1963) and Lindquist (1966), astrophysical relativistic transfer received wide attention (see e.g. Mihalas \& Mihalas 1984 for references to earlier papers). It was realized long ago (see Castor 1972, Mihalas 1980 and references therein) that, for relativistic flows, the interaction between matter and radiation is most easily described if the material properties and the radiation field are evaluated in the frame in which the medium is at rest. The comoving frame transfer equation (CTE) has been considered by Mihalas (1980), Hauschildt \& Wehrse (1991) in the framework of special relativity, and by Schmidt-Burgk (1978), Thorne (1981), Schinder \& Bludman (1989) in the general-relativistic case. Different approaches for the solution of the relativistic transfer problem in planar or spherical geometry have been suggested. They can be grouped, schematically, into three wide classes: direct solution of the CTE using discretization techniques, moment expansion and integration of the CTE along characteristic directions.

The solution of the CTE by finite differencing, like in the DOME method (Hauschildt \& Wehrse 1991), works well in geometrically thin layers, but the treatment of extended atmospheres requires a prohibitive number of discrete elements to obtain a fair angular resolution. In the relatively simple case examined by Hauschildt \& Wehrse, the numerical calculations must be performed on supercomputers even for low resolution grids.

The expansion of the specific intensity in spherical harmonics (moments) 
has the main advantage of reducing the dimensionality of the problem since the angular dependence is suppressed. On the other hand the solution of the transfer problem is reconduced to the solution of a recursive system of partial differential equations that must be truncated at a given order, introducing a certain number of closure conditions. This approach is at the basis of the flux limited diffusion theory (FDT) developed by Levermore \& Pomraining (1981) and generalized by Pomraning (1983), Anile \& Sammartino (1989) and Anile \& Romano (1992). Although in the gray case the FDT provides a self-consistent closure function by solving the differential equation for the flux limiter, the extension to the frequency-dependent problem seems far from being obvious. A very sophisticated, general-relativistic version of the moment formalism was presented by Thorne (1981). It is based on an expansion in projected, symmetric, trace-free (PSTF) moments and, upon truncation, the resulting system of equations can be solved introducing the required number of closure conditions. The closure functions must be specified "a priori" and should reproduce the correct asymptotic limits for the radiation moments when free streaming and diffusion are approached. This method has been fruitfully applied to the solution of astrophysical problems with planar or spherical symmetry, both in the gray and in the frequency-dependent case (see e.g. Turolla \& Nobili 1988, Nobili, Turolla \& Zampieri 1993, Zampieri, Turolla \& Treves 1993). While the arbitrariness of the closure functions is not a serious problem in the gray case, where a large number of moments can be used, it becomes a major complication when frequency-dependent transfer is tackled. In fact, to make the numerical solution affordable, only the first two moments can be taken into account, so that the choice of the closures has a non negligible impact on the results. Moreover, the extension to the bidimensional case is very complicated and requires the specification of 12 closure relations, making 
the method unacceptably dependent on the choice of a large number of free functions.

Characteristics methods are based on the fact that the transfer equation is just the Boltzmann equation for the photon distribution function in phasespace (see e.g. Lindquist 1966). The hyperbolic character of the Boltzmann equation implies that the CTE can be always reduced to a single ordinary differential equation along the characteristic rays. The tangent ray method (TRM) developed in a series of papers by Mihalas and coworkers (Mihalas, Kunasz \& Hummer 1975, 1976a, b, Mihalas 1980) uses a semi-characteristic approach in which the integration is performed along the characteristics of the "spatial" part of the differential operator (the tangent rays), while the frequency derivative is treated by means of a standard finite-differences scheme. A fully characteristic method for the solution of the general-relativistic transfer problem has been discussed by Schmidt-Burgk (1978), Schinder (1988) and Schinder \& Bludman (1989). All these investigations dealt with stationary, spherically symmetric space-times, which admit three Killing vectors: the existence of the associated constants of the motion can be used to obtain simple expressions for the characteristic rays. The analysis by Schinder \& Bludman (1989) was actually restricted to a spacetime characterized by a stationary lagrangian line-element, which corresponds to a vanishing eulerian velocity field for the matter configuration; their test models refer, in fact, to a static atmosphere. The work by Schmidt-Burgk (1978), although finalized to accretion onto a Schwarzschild hole, is, to our knowledge, the only example of an exact solution of the CTE taking into account both the effects of dynamics and strong gravity. For their simple mathematical structure, characteristic methods seem to be promising to cope with realistic astrophysical problems. Moreover, they can be quite naturally extended to more than one spatial dimension, the 
major complication coming from the higher number of ODEs that must be solved to compute the characteristic trajectories.

Previous investigations were mainly concerned with the development of efficient methods for the solution of the CTE, assuming rather simple, often "ad hoc", expressions for the emission and absorption coefficients. This approach is completely justified if one is interested in investigating the formation of particular spectral features, like lines or absorption edges. On the other hand, in all situations in which attention is focussed on the continuum, an accurate treatment of all relevant radiative processes becomes important. When dealing with hot plasmas, the dominant radiative processes are nonconservative scattering and bremsstrahlung. Solutions presented by SchmidtBurgk (1978) refers to a hot, magnetized plasma and takes into account scattering absorption and synchrotron absorption/emission; the collisional term in the Boltzmann equation is written using suitable approximations. On the other hand, approaches based on moment expansion (see e.g. Pomraining 1973 and references therein, Thorne 1981, Prasad et al. 1988) do not permit an exact description of anisotropic and non-coherent scattering, usually treated in the Fokker-Planck approximation. A rigorous treatments of the Compton scattering can be found in Kershaw, Prasad \& Beason (1986), Kershaw (1987), Shestakov, Kershaw \& Prasad (1988), but their results are never been included in transfer codes devoted to astrophysical applications.

In the following we discuss a fully characteristic approach to the solution of the transfer equation in its more general form; results are then specialized to stationary, spherically-symmetric or plane-parallel configurations. Particular care will be devoted to a detailed treatment of the source term for an unmagnetized, fully ionized, non-degenerate hydrogen gas. A numerical code is described and applications to accretion onto black holes and neutron stars 
are finally presented.

\section{RADIATIVE TRANSFER}

In this section we consider the characteristic form of the radiative transfer equation in the comoving frame. In subsection a) the CTE and the equations for its characteristic trajectories are derived in the more general case, when no symmetries are present. A particularization to spherically-symmetric configurations is presented in subsection b). Finally in subsection c) the choice of boundary conditions is discussed. In relativistic transfer the radiation field is naturally described by the photon distribution function in the phase-space, $f$, that is related to the specific intensity by $2 f=c^{2} I / h^{4} \nu^{3}$. Geometrized units ( $c=G=h=1$ ) are used throughout and lengths are in units of the gravitational radius $r=2 M$.

\section{a) The Radiative Transfer Equation}

The relativistic transfer equation, written in covariant form, is just the Boltzmann equation for $f(\mathbf{x}, \mathbf{p})$

$$
\frac{d f}{d \xi}=g(\mathbf{x}, \mathbf{p})
$$

where $\mathbf{p} \equiv d \mathbf{x} / d \xi$ is the photon $4-$ momentum, $\xi$ is an affine parameter along the null geodesic and the collisional term $g$ accounts for the interactions between matter and radiation (see e.g. Lindquist 1966, Thorne 1981). The differential operator in equation (1) acts not merely in spacetime but in the full photons phase-space, made up by the spacetime plus the null tangent space at each point along the photon trajectory.

Since $f(\mathbf{x}, \mathbf{p})$ is a relativistic invariant, equation (1) holds in any frame. However, the material properties (e.g. opacity and emission coefficients, scattering cross-section), which enter the expression of the source term $g$, are 
naturally defined with respect to observers who are locally and instantaneously at rest with the matter (LRF). In the following we adopt a fiducial observer comoving with the fluid, which carries a tetrad $\mathbf{e}_{\hat{a}}$ and has 4 -velocity $\mathbf{u} \equiv$ $\mathbf{e}_{\hat{0}}$. If spacetime, matter and radiation share some common symmetries, the orientation of some of the spatial vectors of the tetrad follows in a natural way. For example, in spherical symmetry, as it will be discussed in detail later on, it is convenient to chose $\mathbf{e}_{\hat{1}}$ orthogonal to the $\theta$ and $\phi$ coordinate directions. With respect to the tetrad, the components of the photon 4-momentum are

$$
p^{\hat{a}}=\left(E, E \mu, E\left(1-\mu^{2}\right)^{1 / 2} \cos \Phi, E\left(1-\mu^{2}\right)^{1 / 2} \sin \Phi\right)
$$

where $E$ is the photon energy, $\mu$ is the cosine of the angle between the photon direction and $\mathbf{e}_{\hat{1}}$, and $\Phi$ is the corresponding azimuthal angle, all measured in the LRF. The three quantities $E, \mu$ and $\Phi$ have an immediate physical meaning and they will be used as independent variables (momentum variables) together with the spacetime coordinates $x^{i}$ to tick events on the light-cone of the phasespace. The total derivative in equation (1) can be explicitated as

$$
\begin{aligned}
& \frac{\partial f}{\partial x^{i}} p^{i}+\frac{\partial f}{\partial p^{\hat{a}}} \frac{d p^{\hat{a}}}{d \xi}= \\
& \frac{\partial f}{\partial x^{i}} p^{i}+\frac{\partial f}{\partial E} \frac{d E}{d \xi}+\frac{\partial f}{\partial \mu} \frac{d \mu}{d \xi}+\frac{\partial f}{\partial \Phi} \frac{d \Phi}{d \xi}=g
\end{aligned}
$$

where $p^{i}=p^{\hat{a}} e_{\hat{a}}^{i}$. The variation of $E, \mu$ and $\Phi$ along the photon trajectory can be obtained from the equation of the null geodesic, written in terms of $p^{\hat{a}}$

$$
\frac{d p^{\hat{a}}}{d \xi}+\Gamma_{\hat{b} \hat{c}}^{\hat{a}} p^{\hat{b}} p^{\hat{c}}=0
$$

where $\Gamma_{\hat{b} \hat{c}}^{\hat{a}}=e_{i}^{\hat{a}} e_{\hat{b}}^{j} e_{\hat{c} ; j}^{i}$ are the Ricci rotation coefficients. Recalling the expression of $p^{\hat{a}}$ given in equation (2), we finally get 


$$
\begin{aligned}
& \frac{d E}{d \xi}=-\Gamma_{\hat{b} \hat{c}}^{\hat{O}} p^{\hat{b}} p^{\hat{c}} \\
& \frac{d \mu}{d \xi}=-\frac{1}{E}\left(\Gamma_{\hat{b} \hat{c}}^{\hat{1}}-\mu \Gamma_{\hat{b} \hat{c}}^{\hat{O}}\right) p^{\hat{b}} p^{\hat{c}} \\
& \frac{d \Phi}{d \xi}=-\frac{1}{E\left(1-\mu^{2}\right)^{1 / 2}}\left(\cos \Phi \Gamma_{\hat{b} \hat{c}}^{\hat{3}}-\sin \Phi \Gamma_{\hat{b} \hat{c}}^{\hat{2}}\right) p^{\hat{b}} p^{\hat{c}} .
\end{aligned}
$$

We note that all the information about the spacetime curvature and the flow dynamics are contained in the tetrad field and enter the Boltzmann equation via the tetrad vectors themselves and their local rates of change which appear in the Ricci coefficients.

Equation (3), together with the set (5), is the more general form of the transfer equation and holds for arbitrary flow motions in any given spacetime. In the next subsection we will discuss how the existence of spacetime symmetries implies that the distribution function is independent on some of the phase-space variables, easing the solution of the transfer problem.

\section{b) Transfer in Spherically-symmetric Spacetimes}

Let us consider the more general spherically-symmetric spacetime, described, in spherical coordinates, by the line-element

$$
d s^{2}=g_{00}(r, t) d t^{2}+g_{11}(r, t) d r^{2}+r^{2}\left(d \theta^{2}+\sin ^{2} \theta d \phi^{2}\right)
$$

Spherical symmetry implies that there exist two constants of the motion, $L_{z}$ and $L$, which are related to the components of the photon 4-momentum by $L_{z}=p_{3}=r^{2} \sin ^{2} \theta p^{3}, L^{2}=r^{4}\left[\left(p^{3}\right)^{2} \sin ^{2} \theta+\left(p^{2}\right)^{2}\right]$. These two expressions take a very simple form, and lead to a major simplification in the transfer equation, if the fluid configuration and the radiation field are themselves sphericallysymmetric. In this case the spatial 3 -velocity $\vec{v}$ of the comoving observer, 
measured by the stationary observer $\delta_{0}^{i} / \sqrt{-g_{00}}$, is in the radial direction and the most convenient choice for the tetrad is

$$
\begin{aligned}
& e_{\hat{0}}^{i}=\left(\frac{\gamma}{\sqrt{-g_{00}}}, \frac{\gamma v}{\sqrt{g_{11}}}, 0,0\right) \\
& e_{\hat{\mathrm{1}}}^{i}=\left(\frac{\gamma v}{\sqrt{-g_{00}}}, \frac{\gamma}{\sqrt{g_{11}}}, 0,0\right) \\
& e_{\hat{2}}^{i}=\left(0,0, r^{-1}, 0\right) \\
& e_{\hat{3}}^{i}=\left(0,0,0, r^{-1} \sin ^{-1} \theta\right)
\end{aligned}
$$

where $\gamma=\left(1-v^{2}\right)^{-1 / 2}$. The constants $L_{z}$ and $L$ may be then expressed in terms of the tetrad components $p^{\hat{a}}$ as

$$
\begin{aligned}
& L_{z}=L \sin \Phi \sin \theta \\
& L^{2}=r^{2} E^{2}\left(1-\mu^{2}\right) .
\end{aligned}
$$

In spherical symmetry, the photon distribution function must be independent on both the polar angles $\phi$ and $\theta$. Since, from equation (7a), we have $\Phi=\Phi(\theta)$, it follows that isotropy in coordinate space implies also that $\partial f / \partial \Phi=0$ and the Boltzmann equation reduces to

$$
\frac{\partial f}{\partial t} p^{0}+\frac{\partial f}{\partial r} p^{1}+\frac{\partial f}{\partial E} \frac{d E}{d \xi}+\frac{\partial f}{\partial \mu} \frac{d \mu}{d \xi}=g .
$$

In the further hypothesis that the spacetime is stationary, the existence of a time-like Killing vector provides a third conserved quantity, $p_{0} \equiv-E_{\infty}$, which can be used to obtain a simple expression for the photon energy along each ray in the LRF

$$
E=\frac{E_{\infty}}{y(1+\mu v)}
$$

in the previous expression $y=\gamma \sqrt{-g_{00}}$ is the specific energy of the fluid, as measured by a static observer at infinity. Clearly the differential operator in 
the transfer equation is Pfaffian, so it is always possible to solve the Boltzmann equation along its characteristic directions, i.e. along the photon trajectories in the 7-dimensional phase-space. In the case at hand, these trajectories lie in a 4-dimensional hypersurface and can be obtained solving equations (5a) and (5b) together with

$$
\begin{aligned}
& \frac{d t}{d \xi}=p^{0}=-\frac{E_{\infty}}{g_{00}} \\
& \frac{d r}{d \xi}=p^{1}=\frac{E y}{r_{g}}(\mu+v) .
\end{aligned}
$$

Actually, the existence of the two constants of motion $L$ and $E_{\infty}$ yields analytical expressions for both $\mu$ and $E$, as functions of $r$, along each photon trajectory:

$$
\begin{aligned}
& \mu=\frac{-y^{2} v b^{2} \pm r\left(r^{2}+b^{2} g_{00}\right)^{1 / 2}}{r^{2}+b^{2} y^{2} v^{2}} \\
& E=\frac{b^{2} y^{2} v^{2}+r^{2}}{\left[r^{2} \pm r v\left(r^{2}+b^{2} g_{00}\right)^{1 / 2}\right]} E_{\infty}
\end{aligned}
$$

where the impact parameter $b=L / E_{\infty}$ has been introduced. Due to spherical symmetry, only positive $b$ 's need to be considered, negative values of the angular momentum give exactly the specular picture, so in the following $b^{2}$ will be used as a parameter. It can be easily shown that the plus/minus sign in equations (11) refers to photons for which $\mu+v$ is always positive/negative. This implies, see equation (10b), that the radial coordinate is always increasing/decreasing along the path and that the condition $\mu+v=0$ defines the locus of turning points for the trajectories. This is just a manifestation of aberration: the turning points, in fact, are located where the cosine of the angle between the photon and radial directions, measured by the stationary observer, vanishes.

Specializing to the vacuum Schwarzschild solution, photon trajectories in physical space may be divided into three classes (see e.g. Misner, Thorne \& 
Wheeler 1973): a) those connecting radial infinity with the event horizon, characterized by impact parameters in the range $0 \leq b^{2}<27 / 4$; b) those that are trapped in the region $1 \leq r<3 / 2$ and $\mathrm{c}$ ) those for which it is always $r>3 / 2$. Trajectories of the latter two types have $b^{2}>27 / 4$. The limiting value $b^{2}=27 / 4$ corresponds to the circular photon orbit. The plot of $\mu=\mu(r ; b)$ and of $E / E_{\infty}=\epsilon(r ; b)$ is shown in figures $1 \mathrm{a}$ and $1 \mathrm{~b}$ for a free-fall velocity law, $u^{1}=r^{-1 / 2}$. As can be seen from the the figures, photons starting at the horizon can reach infinity with non-zero energy only if they are emitted exactly in the radial direction $(\mu=1)$ with an infinite energy, while ingoing photons that leave infinity with zero angular momentum reach the horizon halving their initial energy. At large values of $r$ all rays concentrate at $\mu= \pm 1$, as radial streaming is approached. Trajectories with an impact parameter equal to the critical value $b^{2}=27 / 4$ exhibit a saddle point at $r=3 / 2$.

In the following we will concentrate on the case in which both matter and the radiation field are stationary. Under this assumption the distribution function depends only on three variables, $r, E$ and $\mu$, and since it is $E=E(r)$, $\mu=\mu(r)$ (see equations $[11 \mathrm{a}],[11 \mathrm{~b}])$, the radial coordinate itself can serve as a (non-affine) parameter along the null geodesics. The Boltzmann equation can be then integrated in the domain of existence of each photon trajectory. This particular choice appears to be convenient for a number of reasons, although it poses some numerical problems, as it will be discussed later on. First of all, the treatment of boundary conditions is much simpler when the radial coordinate is the independent variable and this avoids also the integration of equation (10b) along with the transfer equation. Moreover, when scattering is taken into account, the source term depends on the integrals of $f$ over angles, which must be evaluated at both constant $r$ and $E$. The knowledge of $f(r)$ avoids the use of spline or other interpolation algorithms, which is time-consuming and would be 
needed in the case of a different parametrization of the photon trajectories. In conclusion, at least for what concerns the radiation field, the transfer problem can be solved integrating numerically the single differential equation

$$
\frac{d f}{d r}=\frac{r_{g}}{y(\mu+v)} \frac{g}{E}
$$

for different values of the two parameters $b$ and $E_{\infty}$.

At variance with what happens using other methods, like for example expansion in PSTF moments, this kind of approach makes a great simplification in the mathematical structure of the problem: in fact, the non-grey problem can be solved without integration of complicated systems of partial differential equations. Moreover, no closure is needed and this formalism gives as result the full radial, frequency and angle dependent solution. As we will discuss in detail in the next section, is just the knowledge of the angular dependence of the distribution function, lost when the moments of $f$ are used as dependent variables, that gives the possibility to use the characteristic rays method to study the Compton scattering in its more general form; this approach naturally preserves the hyperbolic character of the Boltzmann equation.

In this investigation we focus our attention on the calculation of the radiation field and, thus, we restrict our discussion to the case in which velocity, density and temperature profiles are fixed a priori, similarly to what was done by Mihalas (1980) in the special relativistic case and Schinder \& Bludman (1989) in the general relativistic, static case. Clearly, the full solution of the radiation hydrodynamical problem requires the simultaneous integration of the transfer equation together with the Euler, continuity and energy equations that, in turn, depend on the gray mean intensity $J$ and on the gray radiative flux $H$ :

$$
J=\frac{1}{2} \int_{0}^{\infty} d \nu \int_{-1}^{1} I d \mu=\frac{1}{2} \int_{0}^{\infty} d \nu \int_{-1}^{1} f \nu^{3} d \mu
$$




$$
H=\frac{1}{2} \int_{0}^{\infty} d \nu \int_{-1}^{1} I \mu d \mu=\frac{1}{2} \int_{0}^{\infty} d \nu \int_{-1}^{1} f \nu^{3} \mu d \mu
$$

The coupled solution of the transfer and gasdynamical equation poses, therefore, the same difficulty encountered in the integration of the transfer equation alone in presence of scattering. A numerical technique for the solution of the integrodifferential scattering equation is discussed in section 4a. The same method can be applied to the full radiation hydrodynamical problem and an example is presented in section $5 \mathrm{c}$.

\section{c) Boundary Conditions}

Because there is not a one-to-one map between $r$ and $\xi$, equation (12) must be integrated twice for each value of $b^{2}$, in correspondence with the two solutions for $\mu$ and $E$ given by equations (11a), (11b). At the same time, two different boundary conditions for the distribution function $f$ must be imposed, taking into account that the plus (minus) sign in equations (11) corresponds to outgoing (ingoing) trajectories. The boundary condition for ingoing characteristics of type a) is prescribed in the standard way: for a nonilluminated atmosphere, for example, it is just $f=0$ at the outer edge of the integration domain. This is also the only condition required to integrate the transfer equation along all characteristics of type c), since integration can be started at large $r$ with, say, $f=0$ and carried out until the turning point is reached storing the computed value of $f$, which is then used as the initial condition along the outgoing branch of the trajectory. The remaining rays, including characteristics of type b), can be treated much in the same way if there exists a region in the flow where the effective depth $\tau_{\text {eff }}$ becomes larger than unity at any frequency and LTE is attained. In this case, in fact, the required boundary condition is simply $f=B_{\nu}(T) / E^{3}, B_{\nu}(T)$ is the Planck function at temperature $T$, at a radius $\bar{r}$ such that $\tau_{\text {eff }}(\bar{r})>1$. 
Although this is the standard case for stellar atmospheres, including accretion flows onto compact stars, a different situation may arise when dealing with accretion onto black holes: for low values of the accretion rate, for example, the flow is optically thin all the way down to the horizon (see e.g. Nobili, Turolla \& Zampieri 1991). Now a boundary condition for $f$ must be imposed at $r=1$ for rays starting at the event horizon. Since $E$ goes to infinity there, both the distribution function and $g$ must vanish. The product $E(\mu+v)$, however, does not vanish for all outgoing rays at the horizon, so $g=0$ implies also $d f / d r=0$. In order to avoid numerical overflows, integration is started at a radius $r_{i n}$ fractionally larger than unity, with the regularity condition $d f / d r=0$. The two rays with $b^{2}=27 / 4$ are peculiar since they intersect at $r=3 / 2$ (the saddle point) which is also a critical point for equation (12). We still integrate the transfer equation along these particular rays taking as a regularity condition $g=0$ at $r=3 / 2$. Strictly speaking, this condition is exact only in the case in which the effective optical depth is larger than unity at the last photon orbit; in other cases there is no physical reason to ask for thermalization and the value of $f$ may be undetermined. However, since the radial derivative of $f$ diverges at the critical point, we found that, in a finite differences numerical scheme, the solution of the differential equation fast relaxes and the final result is probably not strongly affected by the value of the distribution function at $r=3 / 2$. 


\section{THE SOURCE FUNCTION}

In the following we deal with an unmagnetized, fully ionized, non-degenerate hydrogen gas in which emitters and absorbers are in local thermal equilibrium at a temperature $T$. We consider also the case in which electrons are relativistic $\left(T \gtrsim 5 \times 10^{9} \mathrm{~K}\right)$, and present a fully general treatment of Compton scattering. However, for the sake of simplicity, we focus our attention only on thermal emission and absorption together with scattering from free electrons; other processes, as pair production and double Compton scattering, that may be relevant at such high temperatures, are outside the scope of this paper. In this section physical units are used; $\gamma$ and $\tau$ denote the dimensionless photon energy and electron temperature, both in units of $m_{e} c^{2} ; K_{p}(x)$ is the modified Bessel function of the second kind.

\section{a) Thermal bremsstrahlung}

The source term for spontaneous emission and absorption, including stimulated emission, can be written as

$$
g=\frac{\eta}{4 \pi h c E^{2}}-\chi E f,
$$

where $\eta$ and $\chi$ are the emission and absorption coefficients, measured in the comoving frame. Because of the assumed equilibrium, Kirchhoff law yields:

$$
\frac{\eta}{4 \pi \chi}=B_{\nu}(T)
$$

with $\nu=E / h$. In the medium we are considering, the dominant true emission and absorption processes are electron-proton and electron-electron bremsstrahlung; in the following we will indicate as $\kappa_{f f}$ the correspondent total opacity. The free-free contribution to the source term is then

$$
\frac{g_{f f}}{E}=\varrho \kappa_{f f}\left(\frac{B_{\nu}}{h c E^{3}}-f\right) .
$$


The photon spectrum from bremsstrahlung is usually described in terms of the velocity-averaged Gaunt factor $G$; in the non relativistic regime tables for $G$ have been presented by Karzas \& Latter (1961). However, as discussed by Gould (1980), contributions to the total energy loss rate due both to relativistic corrections in the electron velocity distribution and to electronelectron bremsstrahlung are already of order $10 \%$ at $T \sim 10^{8} \mathrm{~K}$ become as large as $30 \%$ at $T \sim 10^{9} \mathrm{~K}$. Free-free emissivity from a relativistic thermal plasma has been investigated by several authors (see e.g. Alexanian 1968; Quigg 1968; Haug 1975; Gould 1980; Stepney \& Guilbert 1983; Dermer 1984, Dermer 1986). The photon spectrum from $\mathrm{e}-\mathrm{p}$ emission involves a single quadrature over the relative Lorentz factor of the interacting particles $\gamma_{r}$ (see e.g. Dermer 1986)

$$
\eta_{e-p}(\gamma, \tau)=\frac{n_{e} n_{p} c}{\tau K_{2}(1 / \tau)} \int_{1+\gamma}^{\infty} d \gamma_{r}\left(\gamma_{r}^{2}-1\right) \frac{d \sigma_{B-H}\left(\gamma, \gamma_{r}\right)}{d \gamma} \exp \left(-\frac{\gamma_{r}}{\tau}\right)
$$

where $d \sigma_{B-H}\left(\gamma, \gamma_{r}\right) / d \gamma$ is the Bethe-Heitler cross section corrected for the Elwert factor (see e.g. Heitler 1936) and $n_{e}, n_{p}$ are the number density of electrons and protons. The previous expression holds for $\tau \ll m_{p} / m_{e}$, so that protons can assumed to be at rest in the lab-frame.

Electron-electron emissivity is more complicated since now both particles have the same mass and a quadrupole contribution appears. The standard expression involves a five-fold integral of the totally differential cross-section (Haug 1975), but, as shown by Dermer $(1984,1986)$, it can be reduced to a triple integral exploiting the covariance of Haug's formula to evaluate the cross-section in the CM-frame. The final result is

$$
\begin{aligned}
\eta_{e-e}(\gamma, \tau)= & \frac{n_{e}^{2} c}{4 \tau K_{2}^{2}(1 / \tau)} \int_{1}^{\infty} d \gamma_{r} \frac{\left(\gamma_{r}^{2}-1\right)}{\left[2\left(\gamma_{r}+1\right)\right]^{1 / 2}} \int_{0}^{\zeta\left(\gamma_{r}\right)} \frac{d \gamma^{*}}{\gamma^{*}} \frac{d \sigma_{e-e}^{*}\left(\gamma^{*}, \gamma_{r}\right)}{d \gamma^{*}} \times \\
& \exp \left\{-\frac{\left[2\left(\gamma_{r}+1\right)\right]^{1 / 2}}{\tau}\left(\frac{\gamma^{2}+\gamma^{* 2}}{2 \gamma \gamma^{*}}\right)\right\}
\end{aligned}
$$


(see Dermer 1986 for notation).

The numerical evaluation of both $\eta_{e-p}$ and $\eta_{e-e}$ poses no particular problems and has been carried out following Dermer (1986) in the ranges $2 \times 10^{-2} \leq \tau \leq 10,2 \times 10^{-2} \leq h \nu / K T \leq 25.12$. Numerical results for the total Gaunt factor were then fitted with the analytical function (see Stepney \& Guilbert 1983)

$$
G= \begin{cases}(A+B x) \ln (1 / x)+C+D x, & x=h \nu / K T \leq 2.51 \\ \alpha x^{2}+\beta x+\gamma+\delta / x, & x>2.51\end{cases}
$$

deriving, for each $\tau$, the set of coefficients $A, \ldots, \delta$. The Gaunt factor can be then obtained at any value of $\tau$ and $h \nu / K T$ by means of a suitable interpolation/extrapolation. At temperatures below $\sim 10 \mathrm{keV}(\tau \lesssim 0.01)$, the asymptotic limits of Gould (1980) are used for both e-p and e-e emissivity.

\section{b) Electron scattering}

The second important radiative process we consider is scattering from free electrons: we recall that one of the major complications encountered in solving the transfer equation comes from its non-local character. In fact, even limiting to the coherent and isotropic case, the source term is

$$
\frac{g_{e s}}{E}=\varrho \kappa_{e s}\left(j_{\nu}-f\right)
$$

where $\kappa_{e s}$ is the Thomson opacity and

$$
j_{\nu}=\frac{1}{2} \int_{-1}^{1} f(r, \mu, E) d \mu
$$

is the zero-th moment of the distribution function. Allowing for the more realistic case of Thomson scattering, the correspondent cross section has a 
monopole plus a quadrupole angular dependence (see e.g. Chandrasekhar 1960) yielding

$$
\frac{g_{e s}}{E}=\varrho \kappa_{e s}\left[\frac{3}{8}\left[\left(3 j_{\nu}-k_{\nu}\right)-\mu^{2}\left(j_{\nu}-3 k_{\nu}\right)\right]-f\right]
$$

where

$$
k_{\nu}=\frac{1}{2} \int_{-1}^{1} f(r, \mu, E) \mu^{2} d \mu .
$$

The Thomson limit can be assumed to correctly describe electron scattering when the energy exchange in a single collision can be safely ignored. On the other hand, in high temperature regions non-conservative effects and quantum corrections play a fundamental role in shaping the emergent spectrum. The derivation of the general expression for the Compton source term can be found e.g. in Pomraning (1973) and is briefly outlined below, mainly to introduce some basic ideas which will be used later on when the numerical scheme is discussed. With reference to a single scattering, $\vec{n}$ denotes the incident photon direction and $\xi=\vec{n} \cdot \vec{n}^{\prime}$, where primed quantities refer to the scattered photon. For an incident photon energy $\gamma$ and an electron velocity $\vec{v}_{e}$, the Klein-Nishina formula gives the probability of scattering into the energy $\gamma^{\prime}$ and the direction $\vec{n}^{\prime}$

$$
\begin{aligned}
\sigma\left(\gamma \rightarrow \gamma^{\prime}, \vec{n} \rightarrow \vec{n}^{\prime}, \vec{v}_{e}\right)= & \frac{r_{0}^{2}}{2 \gamma \nu \lambda}\left\{1+\left[1-\frac{(1-\xi)}{\lambda^{2} D D^{\prime}}\right]^{2}+\frac{(1-\xi)^{2} \gamma \gamma^{\prime}}{\lambda^{2} D D^{\prime}}\right\} \\
& \times \delta\left[\xi-1+\lambda \frac{D}{\gamma^{\prime}}-\lambda \frac{D^{\prime}}{\gamma}\right],
\end{aligned}
$$

where

$$
D=1-\vec{n} \cdot \vec{v}_{e} / c, \quad D^{\prime}=1-\vec{n}^{\prime} \cdot \vec{v}_{e} / c, \quad \lambda=\left(1-\frac{v_{e}^{2}}{c^{2}}\right)^{-1 / 2},
$$

$r_{0}$ is the classical electron radius and $\delta$ is the Dirac delta function. Integration over the relativistic maxwellian distribution

$$
f_{e}\left(v_{e}\right)=\frac{\lambda^{5} \exp (-\lambda / \tau)}{4 \pi \tau c^{3} K_{2}(1 / \tau)}
$$


gives the Compton Scattering Kernel (CSK)

$$
\begin{gathered}
\sigma\left(\gamma \rightarrow \gamma^{\prime}, \xi, \tau\right)=\frac{3}{16 \pi \gamma \nu} \int d \vec{v}_{e} \frac{f_{e}\left(v_{e}\right)}{\lambda}\left\{1+\left[1-\frac{1-\xi}{\lambda^{2} D D^{\prime}}\right]^{2}+\right. \\
\left.\frac{(1-\xi)^{2} \gamma \gamma^{\prime}}{\lambda^{2} D D^{\prime}}\right\} \delta\left(\xi-1+\lambda \frac{D}{\gamma^{\prime}}-\lambda \frac{D^{\prime}}{\gamma}\right) .
\end{gathered}
$$

Here the CSK is normalized with respect to $\kappa_{e s} \varrho$, which is reciprocal of the Thomson mean free path; the inverse probability, related to the scattering emissivity, can be obtained from the detailed balance condition

$$
\sigma\left(\gamma \rightarrow \gamma^{\prime}, \xi, \tau\right) \gamma^{2} \exp (-\gamma / \tau)=\sigma\left(\gamma^{\prime} \rightarrow \gamma, \xi, \tau\right) \gamma^{\prime 2} \exp \left(-\gamma^{\prime} / \tau\right)
$$

Further integrations over all outgoing photon directions and energies provide the source term appearing in the Boltzmann equation

$$
\begin{aligned}
\frac{g_{C}}{E}= & \kappa_{e s} \varrho \int_{0}^{\infty} d \gamma^{\prime} \int_{4 \pi} d \Omega^{\prime}\left(\frac{\gamma^{\prime}}{\gamma}\right)^{2} \sigma\left(\gamma^{\prime} \rightarrow \gamma, \xi, \tau\right) f\left(r, \vec{n}^{\prime}, \gamma^{\prime}\right)\left[1+\frac{f(r, \vec{n}, \gamma)}{2}\right] \\
& -\kappa_{e s} \varrho \int_{0}^{\infty} d \gamma^{\prime} \int_{4 \pi} d \Omega^{\prime} \sigma\left(\gamma \rightarrow \gamma^{\prime}, \xi, \tau\right) f(r, \vec{n}, \gamma)\left[1+\frac{f\left(r, \vec{n}^{\prime}, \gamma^{\prime}\right)}{2}\right] .
\end{aligned}
$$

Inserting equation (20) into equation (21), the latter can be written in the more compact form

$$
\begin{aligned}
\frac{g_{C}}{E}= & \kappa_{e s} \varrho \int_{0}^{\infty} d \gamma^{\prime}\left[\exp \left(-\frac{\gamma-\gamma^{\prime}}{2}\right)\left(\frac{f}{2}+1\right)-\frac{f}{2}\right] \int_{4 \pi} d \Omega^{\prime} \sigma\left(\gamma \rightarrow \gamma^{\prime}, \xi, \tau\right) f^{\prime} \\
& -\kappa_{e s} \varrho \sigma_{00} f
\end{aligned}
$$

where $f^{\prime}=f\left(r, \vec{n}^{\prime}, \gamma^{\prime}\right)$ and

$$
\sigma_{00}=\int_{0}^{\infty} d \gamma^{\prime} \int_{4 \pi} d \Omega^{\prime} \sigma\left(\gamma \rightarrow \gamma^{\prime}, \xi, \tau\right)
$$

is the zero-th moment of the CSK (Shestakov, Kershaw \& Prasad 1988). In the previous expressions, non-linear terms account for stimulated scattering. 
The general task of computing the moments of the CSK was undertaken by Shestakov et al. (1988). They have shown that by performing the integration over $\gamma^{\prime}$ first and exploiting the $\delta$-function, the expression of the zero-th moment, which is originally a fivefold integral, can be reduced, after a lot of non-trivial algebra, to a single quadrature

$$
\sigma_{00}=\frac{1 / \gamma}{2 K_{2}(1 / \tau)} \int_{0}^{\infty} d z \gamma z \sigma_{0}(\gamma z) \exp \left[-\frac{1}{2 \tau}\left(z+\frac{1}{z}\right)\right]
$$

where:

$$
\begin{aligned}
y \sigma_{0}(y) & =\frac{3}{8 y}\left[\frac{y^{2}-2 y-2}{y} \ln (2 y+1)+\frac{2 y^{3}+18 y^{2}+16 y+4}{(2 y+1)^{2}}\right] \\
& =y\left(1-2 y+\frac{26}{5} y^{2}-\frac{133}{10} y^{3}+\frac{1144}{35} y^{4}-\cdots\right) \quad \text { for }|y|<\frac{1}{2} .
\end{aligned}
$$

The full evaluation of the Compton source term involves a number of very complicated six dimensional integrals of the distribution function weighted by the CSK for each value of $\gamma, \tau, \mu$. Because only discrete values of the distribution function will be available, all the six quadratures should be, in principle, evaluated numerically at each grid-point and this would make the integration of the transfer equation prohibitively time-consuming. However, as discuss by Kershaw, Prasad \& Beason (1986), two of the three integrals in the CSK become analytical if a particular polar axis for projecting the electron velocity is chosen. Moreover, Kershaw (1987) presented an efficient method for calculating the single integral of the CSK over $\gamma^{\prime}$ or $\xi$ and the double integral over both these variables. A detailed discussion of our algorithm for the evaluation of the first addendum in the Compton source term, that is essentially a re-adaptation of Kershaw's method, is presented later on.

Although the treatment we have just described is the more general to handle Comptonization and proved to be reasonably fast, it remains very timeconsuming, so it is useful to have approximated expressions of $g_{C}$ that can be 
used in some regimes. As it is well known, the complicated nature of the CSK has led many authors to model the Boltzmann equation by a diffusion equation in the frequency space. This approach, the Fokker-Planck approximation, was firstly used by Kompaneets (1956) in the limit of small $\gamma$ and $\tau$. Relativistic corrections to the Kompaneets equation can be included modifying the diffusion coefficient, and a number of efforts were devoted to extend its original form (Fraser 1966, as quoted in Pomraning 1973, Cooper 1971). More recently, Prasad et al. (1988) derived an exact analytical expression for the diffusion coefficient that holds for arbitrary values of $\gamma$ and $\tau$, in the assumption of a nearly isotropic radiation field. The main simplification introduced by the Fokker-Planck approximation is that the integral operator in the transfer equation is replaced by an infinite order differential operator that, for small values of $\gamma$ and $\tau$, truncates at a finite order. The method, originally developed for the non-relativistic transfer equation, is based on an expansion of the specific intensity in a Taylor series about $\nu^{\prime}=\nu$. At the first order in $\gamma$ and $\tau$, Fraser's result is

$$
\begin{aligned}
g_{C} E^{2}= & -\kappa_{e s} \varrho(1-2 \gamma) I+\kappa_{e s} \varrho \int_{4 \pi} d \Omega^{\prime} \sum_{n=0}^{3}\left(\frac{2 n+1}{4 \pi}\right) P_{n}(\xi) S_{n} I \\
& -\frac{3 \kappa_{e s} \varrho}{16 \pi} \frac{c^{2}}{h \nu^{3}} \gamma I\left(1-\nu \frac{\partial}{\partial \nu}\right) \int_{4 \pi} d \Omega^{\prime}\left[1-\xi+\xi^{2}-\xi^{3}\right] I^{\prime}
\end{aligned}
$$

where $P_{n}$ is the Legendre polynomial of order $n$ and $S_{n}(n=0, \ldots, 3)$ are second order differential operators (see Pomraning 1973). Using the standard relation

$$
\xi=\mu \mu^{\prime}+\sqrt{1-\mu^{\prime 2}} \sqrt{1-\mu^{2}} \cos \left(\Phi-\Phi^{\prime}\right)
$$


the previous expression can be cast into the form

$$
\begin{aligned}
\frac{g_{C}}{E}= & \kappa_{e s} \varrho\left[A_{1}+\mu A_{2}+\left(1-\mu^{2}\right) A_{3}+\mu\left(3-5 \mu^{2}\right) A_{4}\right]-\kappa_{e s} \varrho f\{1-2 \gamma+ \\
& \left.\tau\left[A_{5}-\mu^{2} A_{6}+\mu\left(3 \mu^{2}-5\right) A_{7}+\mu\left(3-5 \mu^{2}\right) A_{8}\right]\right\} .
\end{aligned}
$$

The quantities $A_{i}$, containing the first four moments of $f^{\prime}$ and their first and second frequency derivatives, are reported in Appendix A. This is the expression of $g_{C}$ needed in the general relativistic transfer equation in Fraser's approximation. We stress that up to now no assumptions have been made about the angular dependence in the energy exchange terms. A further simplification can be introduced if all terms, but $f$, in equation (26) are assumed to be isotropic and are replaced with their zero-th moments. The Compton source term becomes then

$$
\begin{aligned}
\frac{g_{C}}{E}= & \kappa_{e s} \varrho j_{\nu}\left\{1-\gamma+\gamma \frac{\partial \ln J_{\nu}}{\partial \ln \nu}+\tau\left[\frac{\partial^{2} \ln J_{\nu}}{\partial \ln \nu^{2}}+\left(\frac{\partial \ln J_{\nu}}{\partial \ln \nu}\right)^{2}\right.\right. \\
& \left.\left.-3 \frac{\partial \ln J_{\nu}}{\partial \ln \nu}\right]\right\}-\kappa_{e s} \varrho f\left[1-2 \gamma+\frac{1}{m_{e} \nu^{2}} J_{\nu}\left(1-\frac{\partial \ln J_{\nu}}{\partial \ln \nu}\right)\right],
\end{aligned}
$$

where

$$
J_{\nu}=\frac{1}{2} \int_{-1}^{1} I d \mu
$$

is the mean intensity. The approximated expressions (26) and (27) are to be preferred whenever a non-relativistic plasma is considered, since their evaluation is much faster than that of the general source term given by equation (22). Moreover, equation (27) contains far fewer terms than (26), and has the great advantage that all the angular dependence is contained in $f$.

All forms of the Compton source term based on the Fokker-Planck approximation contain both first and second frequency-derivatives of the moments of the distribution function. As noted by Nobili, Turolla \& Zampieri 
(1993), in connection with the system of the first two PSTF moment equations, Compton terms act as singular perturbations, changing the mathematical character of the differential operator that becomes elliptic. As we discuss in detail later on, our numerical code is based on an iterative scheme in which integral terms, together with their derivatives, are treated as forcing terms, the only full-fledged differential operator being the one contained in the Boltzmann equation. On the other hand, the characteristic ray method provides the angular and frequency dependence for $f$ that allows to write the Compton source term in its original form without resorting to the Fokker-Planck approximation. In this case the problem of radiative transfer with comptonization can be solved exactly in any range of energies and optical depths, and the hyperbolic character of the Boltzmann equation is naturally preserved.

\section{THE NUMERICAL METHOD}

In this section we describe in some detail the numerical scheme we have developed for solving the transfer problem. The more general case, which corresponds to spherical flows in a Schwarzschild spacetime, is discussed in

subsection a); in subsection b) a simplified version of the code, for the solution of the full radiation hydrodynamical problem in static, plane-parallel atmospheres is presented; finally subsection c) is devoted to the numerical evaluation of the Compton source term.

a) The spherical case

As it is well known, in a scattering medium, the transfer equation is an integro-differential equation, while it has a simple structure when only true emission-absorption is included; in particular, it reduces to an ODE when written in its characteristic form. This suggests that its solution can be found using an iterative method in which the starting point is just the solution of the 
transfer problem with only free-free processes taken into account. Following this idea, equation (12) has been integrated numerically, with the boundary conditions previously discussed, for a given set of values of the parameters $b^{2}$ and $E_{\infty}$ and with the source term $g=g_{f f}$. This provides the zero-th order approximation, $f^{(0)}(r, \mu, E)$ of the distribution function, that can be used to evaluate the scattering integrals appearing in $g_{e s}$ or $g_{C}$. In the second step, we use the full expression for $g$ to obtain the first order approximation $f^{(1)}(r, \mu, E)$. This is the solution of the transfer equation written in the form

$$
\frac{y(\mu+v)}{r_{g}} \frac{d f^{(1)}}{d r}=\frac{g_{f f}}{E}+\alpha\left[f^{(0)}\right]-\beta\left[f^{(0)}\right] f^{(1)} .
$$

All the expressions of the scattering source term discussed in the previous section can be cast, and have been presented, in this form. In equations (15), (16), (26) and (27) $\beta$ can be immediately identified with the coefficient of $f$; in equation (22) $\alpha$ is the integral term. The scheme is iterated until convergence is reached, improving at each iteration the functionals $\alpha$ and $\beta$ making use of the distribution function computed in the previous step. As a convergence test, we compared each element of the matrix $j_{\nu}$ with its value relative to the previous iteration and stored the maximum relative correction. Cauchy criterion has been applied to verify the convergence of the succession of such corrections.

Equation (12) has been integrated using a a finite differences method originally developed by Nobili \& Turolla (1988), in which the algebraic system is iteratively solved using the Henyey technique for matrix manipulation. The entire radial domain $\left[r_{i n}, r_{\text {end }}\right]$ is divided by $M$ points; rays of type a) are integrated using this grid. For trajectories which exhibit a turning point, the transfer equation is solved on the same mesh, picking up the subset of grid points which cover their region of existence. Although, as we already mentioned, the choice of $r$ as the parameter along the geodesics has a number of advantages, it 
results in a divergent derivative of $f$ at $\mu=-v$. While, for those branches which approach the turning point, this introduces some errors at most in the last few points, trajectories moving away from the turning points may be systematically affected by an inaccurate determination of their boundary condition. However, it should be taken into account that when the optical depth at the turning point is either large or very small, $f$ tends to $B_{\nu} / h c E^{3}$ or remains vanishingly small, independently on the boundary condition for equation (12). Numerical errors, if any, are, then, restricted to rays inverting in regions of moderate optical depth.

The choice of the $b$-grid strongly constraints the final angular resolution of $f$, and requires special care. Let us first assume that a black hole is the central source; in this case the interval $0 \leq b^{2}<27 / 4$ corresponds to ingoing and outgoing trajectories of class a). For these two subclasses, we fix $N_{1}$ and $N_{2}$ values of the impact parameter in such a way to produce an equally-spaced $\mu$-grid, in the range $[-1,1]$, at the critical point $r=3 / 2$. To discretize the range $b^{2} \geq 27 / 4$, we exploit the one-to-one correspondence between $b^{2}$ and the position of the turning points, $r=r_{n}$,

$$
b_{n}^{2}=\frac{r_{n}^{3}}{r_{n}-1}
$$

We fix $N_{3}$ and $N_{4}$ values of $r_{n}$, the first at $r \leq 3 / 2$ and the latter at $r \geq 3 / 2$; the $r_{n}$ 's are just the radii of the spherical shells tangent to the orbits of types b) and c). In such a way, the total number $N$ of $\mu$-points in the interval $-1 \leq \mu \leq 1$ is $r$-dependent, and it is bounded by $N_{1}+N_{2}+1 \leq N \leq N_{1}+N_{2}+2 N_{3}-1$ for $r \leq 3 / 2$ and $N_{1}+N_{2}+1 \leq N \leq N_{1}+N_{2}+2 N_{4}-1$ for $r \geq 3 / 2$. A better angular resolution in all the radial domain can be obtained increasing the number of photon trajectories. In the case the central source is a star of radius $r_{*}$, the $\mu$-gridding works in a very similar way, but the values of $b$ in the range

$$
0 \leq b^{2}<\frac{r_{*}^{3}}{r_{*}-1}
$$


now produce an equally-spaced $\mu$-mesh at the star radius, the $N_{4}$ points refers to $r>r_{*}$ while no trajectory of type b) is present. We have found more convenient to derive the values of the impact parameter starting from the radial coordinate of the turning points, and not vice versa, since in this way the radial extent of the photon trajectories, and hence the integration range of equation (12), is specified without solving the cubic equation (28).

Once the rays are fixed, equation (12) must be integrated for different values of the parameter $E_{\infty}$ along each trajectory. The range of $E_{\infty}$ should be chosen in such a way that, at each value of $r$, we can compute the distribution function in an interval of the local energy, $\left[E_{\min }, E_{\max }\right]$, large enough to cover the interesting portion of the spectrum. The parameter range $\left[\left(E_{\infty}\right)_{\min },\left(E_{\infty}\right)_{\max }\right]$ must be larger than $\left[E_{\min }, E_{\max }\right]$ at any given radius, since both gravity and dynamics act in changing the photon energy along the geodesics. For $r<r_{\text {end }}$, in fact, the energy interval $\left[E_{\min }, E_{\max }\right]$ is actually influenced by some characteristic rays starting at $r_{\text {end }}$ with $E_{\infty}$ in the range

$$
\left(E_{\infty}\right)_{\min }=[y(1+v)]_{r_{\min }} E_{\min } \leq E_{\infty} \leq[y(1-v)]_{r_{\min }} E_{\max }=\left(E_{\infty}\right)_{\max }
$$

In the numerical calculations we have used the dimensionless energy $x=$ $E / K T_{*}$, where $T_{*}$ is a suitable normalization temperature. For later applications, we found more convenient to divide the storage window $\left[x_{\min }, x_{\max }\right]$ by means of $L$ points equally-spaced in $\ln x$; the same grid is maintained at all radii and $f$ is stored at these points as a function of the local dimensionless energy using an interpolation. In the two remaining ranges $\left[\left(x_{\infty}\right)_{\min }, x_{\min }\right]$ and $\left[x_{\max },\left(x_{\infty}\right)_{\max }\right], 2 P$ values of $x_{\infty}$ has been specified. For these values of the energy at infinity, the transfer equation has been integrated only along the trajectories of those photons that, at some $r$, have a local energy within our storage window. Loading the matrix $f\left(r_{i}, \mu_{j}, E_{k}\right)$ is particularly convenient 
since it allows a more direct calculation of the scattering integrals, that are evaluated at both constant $r$ and $E$. All angular integrals can be obtained simply performing a weighted sum of $f$ over the $\mu$-index without any additional scanning of the array or extra interpolations. This has, also, the advantage that the we are free to choose the most suitable numerical scheme to integrate over energies since the rearrangement of the energy points at each radius follows automatically. The numerical evaluation of the frequency-dependent moments of $f$ has, however, to be carried out with some care. In particular, when the optical depth drops below unity and radial streaming is approached, the integration over $\mu$ becomes troublesome and we found more convenient to perform the quadrature over $b^{2}$, using equation (11a). Since the same change of variable works well near the horizon, where outgoing rays concentrate towards $\mu=1$, it has been used in all the radial range. However, because of the divergence of $d \mu / d b^{2}$ where $\mu=-v$, in a small region around this point the original $\mu$-integration was performed at each value of $r$.

\section{b) The Static, plane-parallel case}

The numerical scheme we have just presented allows the solution of the transfer equation along the geodesic rays in the more general case, when gravity, dynamics and sphericity are all accounted for. In many astrophysical applications, however, transfer of radiation through a static, geometricallythin atmosphere is of interest, like, for example, when studying reprocessing of thermal radiation in the atmosphere of $\mathrm{X}$-ray bursting neutron stars. In all these cases, a plane-parallel approach to the solution of the transfer problem is fully justified since the atmospheric scale height is much less than the star radius, although the effects of the strong gravitational field must still be considered. The assumption of hydrostatic equilibrium introduces a major 
simplification in the treatment of radiative transfer because advection and aberration are no more present. For a vanishing velocity field, equation (9) reduces to $E=E_{\infty} / \sqrt{-g_{00}}$, implying that the value of the local energy at a given radius is the same along all rays. This is just another way of stating the existence of Thorne's (1981) Universal Red-shift Function. The rays are now symmetrical with respect to the $\mu=0$ line. A further, drastic, simplification follows if it can be assumed that the radial coordinate is constant in the atmosphere and equal to the star radius. This is commonly done in non-relativistic transfer theory, replacing the height above the base of the atmosphere with the optical depth. The rays are just straight lines, $\mu=$ const, while the photon energy seen at infinity is simply the energy at any depth redshifted by the constant factor $\left(1-1 / r_{*}\right)^{1 / 2}$. In the light of these considerations, we have developed and tested a reduced version of our code which uses the scattering depth as the independent variable. The angular mesh is obtained specifying directly the values of $\mu$; the energy points at which $f$ is computed coincide with the energy grid, which is the same at all depths. The calculation proceeds exactly in the same way as in a non-relativistic problem and the spectrum at infinity is simply obtained by applying the gravitational red-shift factor to the spectrum emerging at the top of the atmosphere.

An application to isolated neutron stars accreting at low rates is presented in section 5b. In this problem electrons are far from being relativistic so Comptonization can be safely treated in the diffusion approximation using expression (27) for the scattering source term. The much shorter computational time allowed us to solve also the thermal and pressure structure of the atmosphere, coupling the hydrostatic balance and the radiative energy equilibrium to the transfer equation. The hydro equations are solved iteratively, exploiting the scheme for the computation of the scattering integral we have 
already discussed. Pressure and temperature profiles are computed at each iteration step, once the frequency-integrated moments have been obtained.

\section{c) Numerical evaluation of the Compton source term}

As discussed in section 3, the Compton source term, in the form (22), is the sum of two contributions. The second addendum, which requires the calculation of the zero-th moment of the CSK, $\sigma_{00}$, poses no problems since it involves a single quadrature of an analytical function. As proposed by Shestakov et al. (1988), upon the change of variable

$$
u=\frac{1}{\sqrt{2 \tau}}\left(\sqrt{z}-\frac{1}{\sqrt{z}}\right)
$$

$\sigma_{00}$ can be efficiently evaluated using a Gauss-Hermite quadrature. We have tested that six points give an accuracy better than 3 parts in 1000, sufficient for our purposes.

We are left, then, with the problem of finding a fast algorithm for the numerical calculation of the multiple integral

$$
\int_{0}^{\infty} d \gamma^{\prime}\left[\exp \left(-\frac{\gamma-\gamma^{\prime}}{2}\right)\left(\frac{f}{2}+1\right)-\frac{f}{2}\right] \int_{4 \pi} d \Omega^{\prime} \sigma\left(\gamma \rightarrow \gamma^{\prime}, \xi, \tau\right) f^{\prime}
$$

First of all, we note that the scattering probability may become strongly peaked; in the Thomson limit, for example, the CSK tends toward a $\delta$-function at $\gamma=\gamma^{\prime}$. In all regimes in which the integrand is fastly-varying particular care must be used to account for delicate cancellations between opposite terms. We start considering the CSK itself. As discussed by Kershaw, Prasad and Beason (1986), the complicated three-dimensional integral in the electron velocity space can be reduced to a single integral when the solid angle element is defined with respect to a particular polar axis. In fact, taking the polar axis in the direction of the photon momentum transfer $\vec{s}=\left(\gamma^{\prime} \vec{n}^{\prime}-\gamma \vec{n}\right) / q, q=\sqrt{\gamma^{2}+\gamma^{\prime 2}-2 \gamma \gamma^{\prime} \xi}$, 
and using the Dirac $\delta$-function to integrate over the polar angle, the integration over the azimuthal angle becomes analytical. The final form of the CSK is then

$$
\begin{aligned}
& \sigma\left(\gamma \rightarrow \gamma^{\prime}, \xi, \tau\right)=\frac{3}{32 \gamma \nu \tau K_{2}(1 / \tau)} \exp \left(-\lambda_{+} / \tau\right)\left\{\frac{2 \gamma \gamma^{\prime} \tau}{q}\right. \\
& +\int_{\lambda_{+}}^{\infty} \exp \left(-\frac{\lambda-\lambda_{+}}{\tau}\right)\left\{\frac{1}{(1-\xi)^{2}}\right. \\
& \times\left[\frac{(\lambda+\gamma)\left(1 / \gamma+1 / \gamma^{\prime}\right)-(1+\xi)}{\left[(\lambda+\gamma)^{2}+\omega^{2}\right]^{3 / 2}}+\frac{\left(\lambda-\gamma^{\prime}\right)\left(1 / \gamma+1 / \gamma^{\prime}\right)+(1+\xi)}{\left[\left(\lambda-\gamma^{\prime}\right)^{2}+\omega^{2}\right]^{3 / 2}}\right] \\
& +\left[-\gamma \gamma^{\prime}+\frac{2}{1-\xi}+\frac{2}{\gamma \gamma^{\prime}(1-\xi)^{2}}\right] \\
& \left.\left.\times\left[\left[(\lambda+\gamma)^{2}+\omega^{2}\right]^{-1 / 2}-\left[\left(\lambda-\gamma^{\prime}\right)^{2}+\omega^{2}\right]^{-1 / 2}\right]\right\} d \lambda\right\}
\end{aligned}
$$

where the Lorentz factor $\lambda$ is now the integration variable, $\omega^{2}=(1+\xi) /(1-\xi)$ and

$$
\lambda_{+}=\frac{\gamma^{\prime}-\gamma}{2}+\left\{\left[1+\gamma \gamma^{\prime} \frac{1-\xi}{2}\right]\left[1+\frac{\left(\gamma-\gamma^{\prime}\right)^{2}}{2 \gamma \gamma^{\prime}(1-\xi)}\right]\right\}^{1 / 2}
$$

As stressed by Kershaw et al., the main features of the scattering probability are contained in the $\exp \left(-\lambda_{+} / \tau\right) / q$ term: everything is smoothly varying with respect to this quantity, in particular with respect to the exponential. Kershaw et al. proposed two methods for the numerical evaluation of the $\lambda$-integral in equation (30); in both cases the CSK is reduced to an approximate analytical expression. Here we adopt their fastest, although less accurate, algorithm which is based on a suitable division of the integration domain into subintervals where the exponential is replaced by a linear interpolation. To avoid delicate cancellations when $\tau \rightarrow 0$, a Taylor expansion of the inner expression in curly brackets is used to obtain an asymptotic series in terms of Legendre polynomials for the integral; only 
terms up to second order are retained. Using this method the evaluation of the CSK becomes analytical with an accuracy of about 3 parts in a thousand in all parameter ranges. The CPU time for a single evaluation is typically few microseconds on an alpha DEC-3000.

The algorithm we adopt for computing integrals involving the CSK follows the original method presented by Kershaw (1987) for evaluating the total scattering cross-section and it is based on the fact that $\lambda_{+}$has a minimum in both $\gamma^{\prime}$ and $\xi$. The most important contribution to the CSK comes, in fact, from regions near this minimum; everywhere else the scattering probability goes to zero exponentially fast with an $e$-folding length that is simply $\tau$ in $\lambda_{+}$. Having these considerations in mind, the double angular integral in expression (29) can be written, taking $\xi$ and $\bar{\phi}$ as the polar and azimuthal angles, as

$$
\int_{4 \pi} d \Omega^{\prime} \sigma\left(\gamma \rightarrow \gamma^{\prime}, \xi, \tau\right) f^{\prime}=\int_{-1}^{1} d \xi \sigma\left(\gamma \rightarrow \gamma^{\prime}, \xi, \tau\right) \int_{0}^{2 \pi} d \bar{\phi} f\left(r, \mu^{\prime}, \gamma^{\prime}\right)
$$

For each value of $r, \mu, \gamma^{\prime}, \xi$ the azimuthal integral is evaluated using a Lobatto quadrature. The values of the distribution function at

$$
\mu_{l}^{\prime}=\mu \xi+\sqrt{1-\mu^{2}} \sqrt{1-\xi^{2}} \cos \bar{\phi}_{l}
$$

where $\bar{\phi}_{l}$ are the Lobatto abscissae, are obtained from a linear interpolation. Once this is done, for each value of $\gamma, \gamma^{\prime}, \tau$, the integration over all polar directions is carried out picking up the $\xi$ range, within the interval $|\xi| \leq 1$, that provides a non-negligible contribution to the scattering probability: as we anticipated, this is the region around the minimum of $\lambda_{+}$. For fixed $\gamma, \gamma^{\prime}$ and $\tau$, the $e$-folding lengths in $\lambda_{+}, n \tau$, immediately provide the $e$-folding lengths in $\xi, \xi_{n}$. Denoting, in fact, with $\xi_{m}=1-\left|\gamma-\gamma^{\prime}\right| /\left(\gamma \gamma^{\prime}\right)$ the value of $\xi$ where $\lambda_{+}$is minimum, $\xi_{n}$ is the root of the equation

$$
\lambda_{+m 1}+n \tau=\lambda_{+}\left(\gamma, \gamma^{\prime}, \xi_{n}\right)
$$


where $\lambda_{+m 1}=\lambda_{+}\left(\gamma, \gamma^{\prime}, \xi_{M}\right)$ and

$$
\xi_{M}=\max \left(\min \left(\xi_{m},-1\right), 1\right) .
$$

Within each $e$-folding interval we use a 4 -points Lobatto quadrature and the number of intervals is fixed by the request that either the fractional contribution of the last $e$-folding is less than the desired accuracy $\left(5 \times 10^{-3}\right.$ in the present case) or the boundary $\xi= \pm 1$ is reached. At $\gamma=\gamma^{\prime}$ and $\xi=1$ the CSK has an integrable $(\sim \sqrt{1-\xi})$ singularity, that can be easily eliminated with the change of variable $\eta=\sqrt{1-\xi}$.

The integration in energy is carried out in a similar way. Since the most important contribution to the inner integral (over $\xi$ in our scheme) comes from regions where $\lambda_{+}$is near $\lambda_{+m 1}$, the larger contribution to the outer integral (over $\gamma^{\prime}$ ) is provided by regions where $\lambda_{+m 1}$ itself is minimum. Clearly, the lowest values of $\lambda_{+m 1}$ correspond to $\xi_{M}=\xi_{m}$, i.e. to $\xi_{m} \geq-1$; the inequality $\xi_{m} \leq 1$ is always satisfied. We distinguish two cases: for $\gamma^{\prime} \leq \gamma$ the previous condition is verified in the interval

$$
\frac{\gamma}{1+2 \gamma} \leq \gamma^{\prime} \leq \gamma
$$

that we call region $\mathrm{A}$, while for $\gamma^{\prime} \geq \gamma$ it holds in two different domains, that we call in general region $\mathrm{B}$, depending on the value of $\gamma$ :

$$
\begin{aligned}
\gamma \leq \gamma^{\prime} \leq \frac{\gamma}{1-2 \gamma} \quad \text { if } \quad \gamma<1 / 2 \\
\gamma \leq \gamma^{\prime}<\infty \quad \text { if } \quad \gamma \geq 1 / 2 .
\end{aligned}
$$

Since in region $\mathrm{A} \lambda_{+m 1}=1$, the search for the $e$-folding lengths is not required and integration is straightforward. This is not the case in region $\mathrm{B}$, where $\lambda_{+m 1}=1+\gamma^{\prime}-\gamma$. Now, although its minimum value is still $\lambda_{+m 2}=1, \lambda_{+m 1}$ 
is not a constant. The corresponding $e$-folding lengths $\gamma_{n}^{\prime}$ are to be derived solving the equation

$$
\lambda_{+m 2}+n \tau=\lambda_{+}\left(\gamma, \gamma_{n}^{\prime}, \xi_{M}\right)
$$

which reduces to

$$
1+n \tau=1+\gamma_{n}^{\prime}-\gamma
$$

and gives simply $\gamma_{n}^{\prime}=\gamma+n \tau$. In region $\mathrm{B}$ integration over $\gamma^{\prime}$ is carried out using the same procedure introduced for the $\xi$-quadrature. To complete our discussion, we need to consider the two intervals

$$
0 \leq \gamma^{\prime}<\frac{\gamma}{1+2 \gamma}
$$

region $\mathrm{C}$, and, if $\gamma<1 / 2$,

$$
\frac{\gamma}{1-2 \gamma} \leq \gamma^{\prime}<\infty
$$

region D. In both cases $\lambda_{+m 1}=\lambda_{+}\left(\gamma, \gamma^{\prime},-1\right)$ and its minimum is reached at

$$
\lambda_{+m 2}=\left.\lambda_{+m 1}\right|_{\gamma^{\prime}=\gamma /(1+2 \gamma)}
$$

or

$$
\lambda_{+m 2}=\left.\lambda_{+m 1}\right|_{\gamma^{\prime}=\gamma /(1-2 \gamma)}
$$

in regions $\mathrm{C}$ and $\mathrm{D}$ respectively. The corresponding $e$-folding lengths are obtained from equation (36). Lobatto rule is used everywhere and stepping is terminated when its fractional contribution becomes less than $5 \times 10^{-3}$. The most convenient number of Lobatto points depends on the typical relative values of the photon energy and gas temperature. In fact, for different values of $\gamma$ and $\tau, \lambda_{+}\left(\gamma^{\prime}\right)$ can be either strongly peaked or very broad near its minimum. Optimization requires some numerical experimenting, looking for the best agreement between the direct evaluation of the CSK double integral and $\sigma_{00}$ 
computed using equations (24). For the test model presented in subsection 5b, we used either a six or a ten points quadrature. Accordance between the values of $\sigma_{00}$ obtained using the two methods is better than $3-4 \%$, with the larger errors in the external region where the radiation temperature (mean photon energy) is very far away from the gas temperature. On the other hand, where the Compton parameter $Y_{C}$ (see e.g. Rybicki \& Lightman 1979) is greater than unity, accuracy is better than 7 parts in a thousand. We finally note that the choice of a gaussian-type quadrature is motivated, basically, by the fact that we need to perform integrals of the CSK times $f$. The distribution function must be interpolated to obtain its values at the integration points. Clearly, gaussiantype quadratures with a fixed number of abscissae are much faster, although less accurate, than step-adaptive schemes, as the Simpson rule originally used by Kershaw. Computational feasibility is also the reason for which we decided to evaluate the integral (29) using the values of $f$ relative to the previous iteration. Clearly, it is possible to rewrite expression (29) as

$$
\begin{aligned}
& \frac{f}{2} \int_{0}^{\infty} d \gamma^{\prime}\left[\exp \left(-\frac{\gamma-\gamma^{\prime}}{2}\right)-1\right] \int_{4 \pi} d \Omega^{\prime} \sigma\left(\gamma \rightarrow \gamma^{\prime}, \xi, \tau\right) f^{\prime} \\
& +\int_{0}^{\infty} d \gamma^{\prime} \exp \left(-\frac{\gamma-\gamma^{\prime}}{2}\right) \int_{4 \pi} d \Omega^{\prime} \sigma\left(\gamma \rightarrow \gamma^{\prime}, \xi, \tau\right) f^{\prime}
\end{aligned}
$$

since $f$ does not depend on $\gamma^{\prime}$. Now $f$ is just the dependent variable of the transfer equation at any iterative step. The drawback is that the computing times is about doubled, because of the two multiple integrals. The CPU time for a single evaluation of expression (29) is typically $\sim 0.1 \mathrm{~s}$ and, in an production run, a $\sim 2 \times 10^{5}$ evaluation are required, implying a total time of about $6 \mathrm{hr}$. 


\section{APPLICATIONS}

As we stressed several times, accretion flows onto compact objects, and black holes in particular, provide an ideal arena for applications of relativistic radiation transfer in differentially-moving media. The accreting matter reaches, in fact, not only $r \sim 1$ with $v \sim 1$, but, often, temperatures high enough $\left(T \gtrsim 10^{9} \mathrm{~K}\right)$ to make a full treatment of Comptonization necessary. For this reason we decided to present in this section the numerical solutions of the transfer problem relative to different accretion regimes onto black holes and neutron stars: "cold" and "hot" black hole accretion is considered in subsections a) and b), respectively, and subsection c) deals with "cold", static atmospheres around neutron stars. The full radiation hydrodynamical problem in solved only in the latter case, while in the first two examples the flow hydrodynamics is kept fixed. Since our present goal is to test the capabilities of our method, no attempt has been made to explore the models parameter space: we just present results for a single model which we judge useful in illustrating the main features of our integration scheme. For model c) a direct comparison with the results obtained by Zampieri et al. (1995) with the moment expansion has been made, showing a good agreement. No previous solutions for black hole accretion spectra are available, at least for models which contain an optically thick core. Our attempt to cross-check results presented in section 5a integrating the moment equations were hindered by severe numerical problems which arise when the flow is not effectively thick at the horizon at all frequencies. The moment method can not, also, be used to compute radiative transfer in "hot" models, where Compton scattering must be treated outside the Fokker-Planck approximation.

\section{a) Accretion onto black holes: low-luminosity solutions}

Spherical, stationary accretion onto a Schwarzschild black hole has been 
throughly investigated in the past and we refer to the paper by Nobili, Turolla and Zampieri (1991, NTZ in the following) for all details. A distinctive feature of black hole accretion is that, for the same value of the accretion rate $\dot{m}$ which is the only free parameter, two solutions may exist with very different properties: a "cold", low-luminosity and a "hot", high-luminosity one. In both solutions the sonic point is so far away that we can safely assume that matter is free-falling with $u^{1}=r^{-1 / 2}$ in our region of interest. Here we refer to models with high accretion rates, $\dot{m} \gtrsim 1$ in Eddington units. In this regime low-luminosity solutions start to develop an inner region optically thick to both free-free and scattering and show negligible Comptonization; consequently, electron scattering can be treated in the Thomson limit, using expression (16). Under these conditions we expect, however, bulk motion Comptonization in the converging flow (Blandford \& Payne 1981; Payne \& Blandford 1981; Nobili, Turolla \& Zampieri 1993) to act efficiently at high frequencies where true absorption is very low.

For our first test we consider NTZ solution characterized by $\dot{m}=0.71$, corresponding to a density at the horizon $\varrho_{H}=10^{-6} \mathrm{~g} \mathrm{~cm}^{-3}$. The gas temperature is in the range $2 \times 10^{4} \mathrm{~K} \lesssim T \lesssim 5 \times 10^{5} \mathrm{~K}$, so we have chosen a normalization temperature $\ln T_{*}=11$. The dimensionless energy window is $x_{\min }=0.1<x<x_{\max }=40$, corresponding to the range $0.5-206 \mathrm{eV}$; here $L=30$ points have been used. Outside this range, $P=10$ energies has been fixed in each of the two additional intervals of $x_{\infty}$ we need, as discussed in section 3a. Since the effective optical depth at our larger energies is everywhere $<1$, we solved the transfer problem for $10^{-2} \leq \log r \leq 5$, imposing the boundary condition $d f / d r=0$ along trajectories starting at $r_{i n}$. The radial domain has been divided by $M=250$ points; the grid is not uniformly spaced and points are tighter around $r=3 / 2$. To obtain a good angular resolution, 90 trajectories 
have been followed at each energy, $N_{1}=N_{2}=20, N_{3}=10, N_{4}=40$. In such a way, the number of $\mu$ points, which is minimum at $r=3 / 2$, is always greater than 21. At each value of $x$ within the storage window the scattering source term was calculated using a linear interpolation for both the matrices $j_{\nu}$ and $k_{\nu}$; outside this window an extrapolation has been used. Figures $2 \mathrm{a}$ and $2 \mathrm{c}$ show the mean intensity $J_{\nu}$ and radiation pressure $K_{\nu}$ at different energies, together with the Planck function at $T\left(r_{i n}\right)$; each curve corresponds to a different value of the radial coordinate. The effective frequency-dependent optical depth goes from $3 \times 10^{3}$ to $10^{-4}$ for the lowest frequency, while high energy photons stream freely at all radii. The low energy portion of the spectral distribution is a superposition of thermal bremsstrahlung emission at different temperatures while bulk motion Comptonization produces a power law highenergy tail. The calculated spectral index, $\alpha=-2.9$, is due to unsaturated bulk motion comptonization, being the scattering optical depth $\sim 0.7$ at the horizon. The theoretical value derived by Payne \& Blandford, in the limit $\tau_{e s} \gg 1$, is $\alpha=-2$ for a free-fall velocity. At large radii, where radial streaming is approached, all moments fall off as $r^{-2}$; the asymptotic radial gradient we have found is -1.99 . While the evaluation of even moments does not pose particular problems, in the inner regions, where the radiation field is nearly isotropic, a direct numerical quadrature for computing odd moments becomes troublesome because of the delicate cancellations between contributions of opposite sign. To avoid this problem, the monochromatic flux, presented in figure $2 \mathrm{~b}$, has been replaced with its analytical expression in the diffusion approximation every time it is $\tau_{\text {eff }}>10$. A typical production run required 10-11 iterations to converge with a fractional accuracy better than $10^{-4}$, with a total CPU time of about 20 minutes on an alpha DEC-3000.

\section{b) Accretion onto black holes: high-luminosity solutions}


In "hot" solutions temperature is much higher, typically $\sim 10^{10} \mathrm{~K}$ near to the horizon. As a consequence, free-free absorption is much lower than in "cold" models, even for larger accretion rates. Along the high-luminosity branch, thermal Comptonization is the dominant radiative process and it must be treated in its more general form, using expression (22). Here we consider the "hot" solution of NTZ with $\dot{m}=71, \varrho_{H}=10^{-4} \mathrm{~g} \mathrm{~cm}^{-3}$. The flow is now effectively thin at all frequencies, although an inner core optically thick to scattering is present. The gas temperature in this model is in the range $10^{5} \mathrm{~K} \leq T \leq 10^{10} \mathrm{~K}$, so we have chosen $\ln T_{*}=21$ and $x_{\min }=0.008$. Now the energy window is $0.9-4500 \mathrm{keV}$ and $L=35$ points have been used. Since the evaluation of the CSK integrals is very time-consuming, both angular and radial resolution has been reduced with respect to the previous model: $N_{1}=$ $N_{2}=N_{3}=10, N_{4}=30$ and $M=110$ in the same radial domain. In this model convergence has been reached with a fractional accuracy better than 0.02 , and the calculated radial gradient at infinity is -2.04 . The resulting mean intensity is presented in figure 3. In high temperature models, the mean intensity is always less than $B_{\nu}$, but despite the accreting gas radiates less efficiently than in low-luminosity optically thick solutions, the efficiency of accretion process is higher, due to the fact that the matter temperature is now higher in the whole photospheric region. Since the emergent spectrum is peaked at about 40 $\mathrm{keV}$, these solutions, if stable (see NTZ and Zampieri, Miller \& Turolla 1995), seem to provide a natural way to produce hard X-ray radiation with reasonable efficiency out of spherical accretion onto black holes.

c) Static, plane-parallel atmospheres around neutron stars

Our last application refers to a static, "cold" atmosphere around an accreting unmagnetized neutron star. Emitted spectra were firstly derived by 
Zel'dovich \& Shakura (1969) and, in more detail, by Alme \& Wilson (1973) in the high luminosity range $\left(l \gtrsim 10^{-3}\right.$ in Eddington units). Solutions for $10^{-7}<l<10^{-3}$ has been recently presented by Zampieri et al. (1995) in connection with isolated neutron stars accreting the interstellar medium. As they have shown, the emitted spectrum exhibits an overall hardening with respect to the blackbody at the neutron star effective temperature with a hardening ratio, typically $\sim 1.5-3$, which increases with decreasing luminosity. The most important physical processes are free-free emission and absorption; Compton cooling plays a role only in increasing the temperature in the outer atmospheric layers, where the low energy tail of the emitted spectrum is created. Since typical temperatures are very low, Comptonization is treated by means of the approximated expression (27), which is much faster. The run of thermodynamical variables is obtained solving the hydrostatic and energy balance (see Zampieri et al.) together with the transfer equation, using an iterative scheme. However, in this problem the thermal balance is very delicate and the zone where photons of different energies thermalize strongly depends on integrated quantities, $J$ and the absorption mean $\kappa_{0}$. Numerical integration proved more stable if $J$ and $H$ are derived as solution of the first two gray moment equations. The same approach was used by Zampieri et al., with the difference that in our scheme the gray moment equations are solved exactly, computing from the specific intensity the Eddington factors $K / J$ at each depth and $J / H$ at $\tau=0$. Here we have recomputed the model with $l=10^{-4}$, using a spectral window $x_{\min }=0.1, x_{\max }=10$ centered around a normalization temperature $\log T_{*}=6.6 ; L=30$ frequency points have been used. The angular resolution is provided by $N_{1}=N_{2}=15$ trajectories and the transfer equation is solved in the range $-8<\log (\tau)<0.9$ using $M=100$ grid points. The resulting mean intensity is plotted in figure 4 . Figures 5 and 6 show the emergent 
spectrum and the temperature profile together with the results of Zampieri et al. (dashed lines). As can be seen, the agreement both in the spectral shape and the temperature profile is very good, showing that the approximated solution of the transfer equation with two moments is rather accurate. To obtain this model, with a fractional accuracy better than $2 \times 10^{-2}$, 14 iterations were required, with a total CPU time of about 3 minutes on an alpha DEC-3000. Agreement between the gray mean intensity, derived as the double integral of $f$, and the solution of the second gray moment equation is always better than few parts in thousand.

\section{CONCLUSIONS}

In this paper we have presented a characteristic method for the solution of the general relativistic transfer equation. If the spacetime admits some symmetries, the formalism can be simplified; in particular, in presence of three Killing vectors, two of the three equations for the characteristic rays become analytical. In addition, using the radial coordinate as the parameter along the null geodesics, the exact solution of the transfer problem can be obtained solving a single ordinary differential equation along a bi-parametric family of characteristic trajectories. A numerical technique, based on an iterative scheme, has been developed and tested either for the calculation of the radiation field in a fixed background or for the solution of the full radiation hydrodynamical problem in spherical and plane-parallel geometry. Particular care has been devoted to the evaluation of the source term, taking into account radiative processes which are believed to be of importance in astrophysical accreting plasmas: electron-electron and electron-proton bremsstrahlung, Thomson and Compton scattering.

Radiative effects due to magnetic fields and pair production-annihilation 
were not considered in this work. However we stress that the method we have presented is completely general and additional radiative processes may be easily included. Source terms not involving integrals of the photon distribution function can be simply accounted for when the corresponding emissivity and opacity coefficients are provided. On the other hand, our iterative scheme allows for the solution of integro-differential equations and can be used to include also different integral source terms as, for instance, those ones related to pair production or bound-bound emission. Actually, a self-consistent treatment of pair production entails the solution of the full radiation hydrodynamical problem, with the addition of the pair balance equation and was left out on purpose in our discussion of "hot" accretion solutions which are obtained at fixed hydrodynamics.

In the test models we discussed magnetic fields and pair production are not expected to play a relevant role at least for low luminosity solutions. In the case of accretion onto neutron stars it can be easily shown, in fact, that, for typical temperatures and densities in the photospheric region, the cyclotron emission is lower than the free-free emission if $B \lesssim 10^{9} \mathrm{G}$ (see e.g. Schmid-Burgk, 1978). On the other hand, a relic magnetic field of this order is just what is expected in isolated neutron stars which evolved beyond the pulsar phase; our models can be then assumed to describe correctly the emitted spectrum from old neutron stars accreting the interstellar medium. As far as low-luminosity accretion onto black holes is concerned, the limiting value is a factor $10^{-2}$ smaller, but it still exceeds the maximum strength of the tangled $B$-field derived assuming equipartition between magnetic and thermal energy densities. However, in the inner regions of high-luminosity models electrons become relativistic and both pair processes and synchrotron emission start to play a role. 


\section{REFERENCES}

Alexanian, M. 1968, Phys. Rev., 165, 253

Alme, M.L., \& Wilson, J.R. 1973, ApJ, 186, 1015

Anderson, J.L. \& Spiegel, E.A. 1972, ApJ, 171, 127

Anile, A.M., \& Sammartino, M. 1989, Ann. Phys., 14, 325

Anile, A.M., \& Romano, V. 1992, ApJ, 386, 325

Blandford, R.D., \& Payne, D.G. 1981a, MNRAS, 194, 1033

Blandford, R.D., \& Payne, D.G. 1981b, MNRAS, 194, 1041

Castor, J.I. 1972, ApJ, 178, 779

Chandrasekhar, S. 1960, Radiative Transfer, (New York: Dover)

Cooper, G. 1971, Phys. Rev. D, 3, 2312

Dermer, C.D. 1984, ApJ, 280, 328

Dermer, C.D. 1986, ApJ, 307, 47

Gould, R.J. 1980, ApJ, 238, 1026

Haug, E. 1975, Zs. Naturforschung, 30a, 1099

Hauschildt, P.H. \& Wehrse, R. 1991, J. Quantit. Spectros. Radiat. Transfer, 46,81

Heitler, W. 1936, The Quantum Theory of Radiation, (Oxford: Oxford University Press)

Kershaw, D.S., Prasad, M.K., \& Beason, J.D. 1986, J. Quantit. Spectros. Radiat. Transfer, 36, 273

Kershaw, D.S. 1987, J. Quantit. Spectros. Radiat. Transfer, 38, 347

Kompaneets, A.S. 1956, Soviet Phys. JETP, 4, 730

Levermore, C.D., \& Pomraning, G.C. 1981, ApJ, 248, 321

Lindquist, R.W. 1966, Ann. Phys. (NY), 37, 487

Loeb, A., McKee, C.F., \& Lahav, O. 1991, ApJ, 374, 44

Madej, J. 1989, ApJ, 339, 386 
Melia, F., \& Zylstra, G.J. 1991, ApJ, 374, 732

Mihalas, D., Kunasz, P.B., \& Hummer, D.G. 1975, ApJ, 202, 465

Mihalas, D., Kunasz, P.B., \& Hummer, D.G. 1976a, ApJ, 203, 647

Mihalas, D., Kunasz, P.B., \& Hummer, D.G. 1976b, ApJ, 206, 515

Mihalas, D. 1980, ApJ, 237, 574

Mihalas, D., Winkler, K-H., \& Norman, M.L. 1984, J. Quantit. Spectros. Radiat. Transfer, 31, 479

Mihalas, D. \& Mihalas, B. 1984, Foundations of Radiation Hydrodynamics (Oxford: Oxford University Press)

Misner, C.W., Thorne, K.S., \& Wheeler, J.A. 1973, Gravitation, Box 25.7 (San Francisco: Freeman \& Co.)

Nobili, L., \& Turolla, R. 1988, ApJ, 333, 248

Nobili, L., Turolla, R., \& Zampieri, L. 1991, ApJ, 383, 250

Nobili, L., Turolla, R., \& Zampieri, L. 1993, ApJ, 404, 686

Payne, D.G., \& Blandford, R.D. 1981, MNRAS, 196, 781

Pomraning, G.C. 1973, The Equations of Radiation Hydrodynamics (New York: Pergamon Press)

Pomraning, G.C. 1983, ApJ, 266, 841

Prasad, M.K., Shestakov, A.I., Kershaw, D.S., \& Zimmerman, G.B. 1988, J. Quantit. Spectros. Radiat. Transfer, 40, 29

Quigg, C. 1968, ApJ, 151, 1187

Rybicki, G.B., \& Lightman, A.P. 1979, Radiative Processes in Astrophysics (New York: Wiley)

Schinder, P.J. 1988, Phys. Rev. D, 38, 1673

Schinder, P.J. \& Bludman, S.A. 1989, ApJ, 346, 350

Schmid-Burgk, J. 1978, APSS, 56, 191 
Shestakov, A.I., Kershaw, D.S., \& Prasad, M.K. 1988, J. Quantit. Spectros. Radiat. Transfer, 40, 577

Simon, R. 1963, J. Quantit. Spectros. Radiat. Transfer, 3, 1

Stepney, S., \& Guilbert, P.W. 1983, MNRAS, 204, 1269

Thomas, L.H. 1930, Q. Jl. Math., 1, 239

Thorne, K.S. 1981, MNRAS, 194, 439

Turolla, R., \& Nobili, L. 1988, MNRAS, 235, 1273

Zampieri, L., Miller, J.C., \& Turolla, R. 1995, MNRAS, in the press

Zampieri, L., Turolla, R. \& Treves, A. 1993, ApJ, 419, 311

Zampieri, L., Turolla, R., Zane, S. \& Treves, A. 1995, ApJ, 439, 849

Zel'dovich, Ya., \& Shakura, N. 1969, Soviet Astron.-AJ, 13, 175

\section{APPENDIX A}

Here we present the expressions of the $A_{i}, i=1, \ldots, 8$, terms appearing into equation (26). Their derivation starts from Fraser's result, equation (25), and makes use of relation

$$
\xi=\mu \mu^{\prime}+\sqrt{1-\mu^{\prime 2}} \sqrt{1-\mu^{2}} \cos \left(\Phi-\Phi^{\prime}\right) .
$$

At first order in $\gamma$ and $\tau$, only the first four moments $m_{\nu}^{n}$ of the distribution function

$$
m_{\nu}^{n}=\frac{1}{2} \int_{-1}^{1} f \mu^{n} d \mu
$$

appear in the Compton source term; they will be termed $j_{\nu}, h_{\nu}, k_{\nu}$ and $l_{\nu}$, using the standard notation. We introduce also the correspondent moments of the specific intensity $I$ :

$$
M_{\nu}^{n}=E^{3} m_{\nu}^{n} .
$$

To make the larger number of terms dimensionless, we introduce double logarithmic frequency derivatives for all even moments; terms containing odd 
moments are written as semi-logarithmic derivatives since odd moments may become vanishingly small in regions where the effective optical depth is very large. The final result is:

$$
\begin{aligned}
A_{1}= & j_{\nu}\left\{1-\gamma\left(1-\frac{\partial \ln J_{\nu}}{\partial \ln \nu}\right)+\tau\left[\frac{\partial^{2} \ln J_{\nu}}{\partial \ln \nu^{2}}+\left(\frac{\partial \ln J_{\nu}}{\partial \ln \nu}\right)^{2}-3 \frac{\partial \ln J_{\nu}}{\partial \ln \nu}\right]\right\} \\
A_{2}= & \frac{6}{5}\left[(\gamma-\tau) h_{\nu}-\frac{1}{E^{3}}(\gamma-3 \tau) \frac{\partial H_{\nu}}{\partial \ln \nu}-\frac{\tau}{E^{3}} \frac{\partial^{2} H_{\nu}}{\partial \ln \nu^{2}}\right] \\
A_{3}= & \frac{1}{8}\left\{(1-\gamma-6 \tau)\left(j_{\nu}-3 k_{\nu}\right)+(\gamma-3 \tau)\left(j_{\nu} \frac{\partial \ln J_{\nu}}{\partial \ln \nu}-3 k_{\nu} \frac{\partial \ln K_{\nu}}{\partial \ln \nu}\right)\right. \\
& \left.+\tau\left[j_{\nu}\left(\left(\frac{\partial \ln J_{\nu}}{\partial \ln \nu}\right)^{2}+\frac{\partial^{2} \ln J_{\nu}}{\partial \ln \nu^{2}}\right)-3 k_{\nu}\left(\left(\frac{\partial \ln K_{\nu}}{\partial \ln \nu}\right)^{2}+\frac{\partial^{2} \ln K_{\nu}}{\partial \ln \nu^{2}}\right)\right]\right\} \\
A_{4}= & \frac{3}{40}\left[(\gamma+4 \tau)\left(3 h_{\nu}-5 l_{\nu}\right)-\frac{1}{E^{3}}(\gamma-3 \tau)\left(3 \frac{\partial H_{\nu}}{\partial \ln \nu}-5 \frac{\partial L_{\nu}}{\partial \ln \nu}\right)\right. \\
& \left.-\frac{\tau}{E^{3}}\left(3 \frac{\partial^{2} H_{\nu}}{\partial \ln \nu^{2}}-5 \frac{\partial^{2} L_{\nu}}{\partial \ln \nu^{2}}\right)\right] \\
A_{5}= & \frac{3}{8}\left(3 j_{\nu}-k_{\nu}-3 j_{\nu} \frac{\partial \ln J_{\nu}}{\partial \ln \nu}+k_{\nu} \frac{\partial \ln K_{\nu}}{\partial \ln \nu}\right) \\
A_{6}= & \frac{3}{8}\left(j_{\nu}-3 k_{\nu}-j_{\nu} \frac{\partial \ln J_{\nu}}{\partial \ln \nu}+3 k_{\nu} \frac{\partial \ln K_{\nu}}{\partial \ln \nu}\right) \\
A_{7}= & \frac{3}{8}\left(h_{\nu}-\frac{1}{E^{3}} \frac{\partial H_{\nu}}{\partial \ln \nu}\right) \\
A_{8}= & \frac{3}{8}\left(l_{\nu}-\frac{1}{E^{3}} \frac{\partial L_{\nu}}{\partial \ln \nu}\right)
\end{aligned}
$$




\section{FIGURE CAPTIONS}

Figure 1a. The run of the cosine of the angle between the photon momentum and the radial direction, as measured by a free-falling observer, along the characteristic rays. Different curves correspond to different values of the impact parameter $b$.

Figure 1b. Same as in figure 1a for the photon energy normalized with respect to $E_{\infty}$.

Figure 2a. Monochromatic mean intensity at different radii (full lines), together with the blackbody function at $T\left(r_{i n}\right)$ (dashed line), for "cold" accretion onto a black hole with $\dot{m}=0.71$.

Figure 2b. Same as in figure 2a for the monochromatic flux.

Figure 2c. Same as in figure $2 \mathrm{a}$ for the monochromatic radiation pressure.

Figure 3. Monochromatic mean intensity at different radii (full lines), together with the corresponding blackbody function at $T\left(r_{i n}\right)$ (dashed line), for "hot" accretion onto a black hole with $\dot{m}=71$.

Figure 4. Monochromatic mean intensity at different scattering depths (full lines) for a "cold", static, plane-parallel atmosphere around a neutron star with $l=10^{-4}$. The blackbody function at $T\left(\tau_{i n}\right)$ is also drawn for comparison (dashed line).

Figure 5. The emergent spectrum for the model in figure 4 (full line) compared with the blackbody at the neutron star effective temperature (dash-dotted line) and with the solution obtained by Zampieri et al. (1995, dashed line).

Figure 6. The gas temperature profile for the model in figure 4 (full line), compared with that one found by Zampieri et al. (1995, dashed line). 


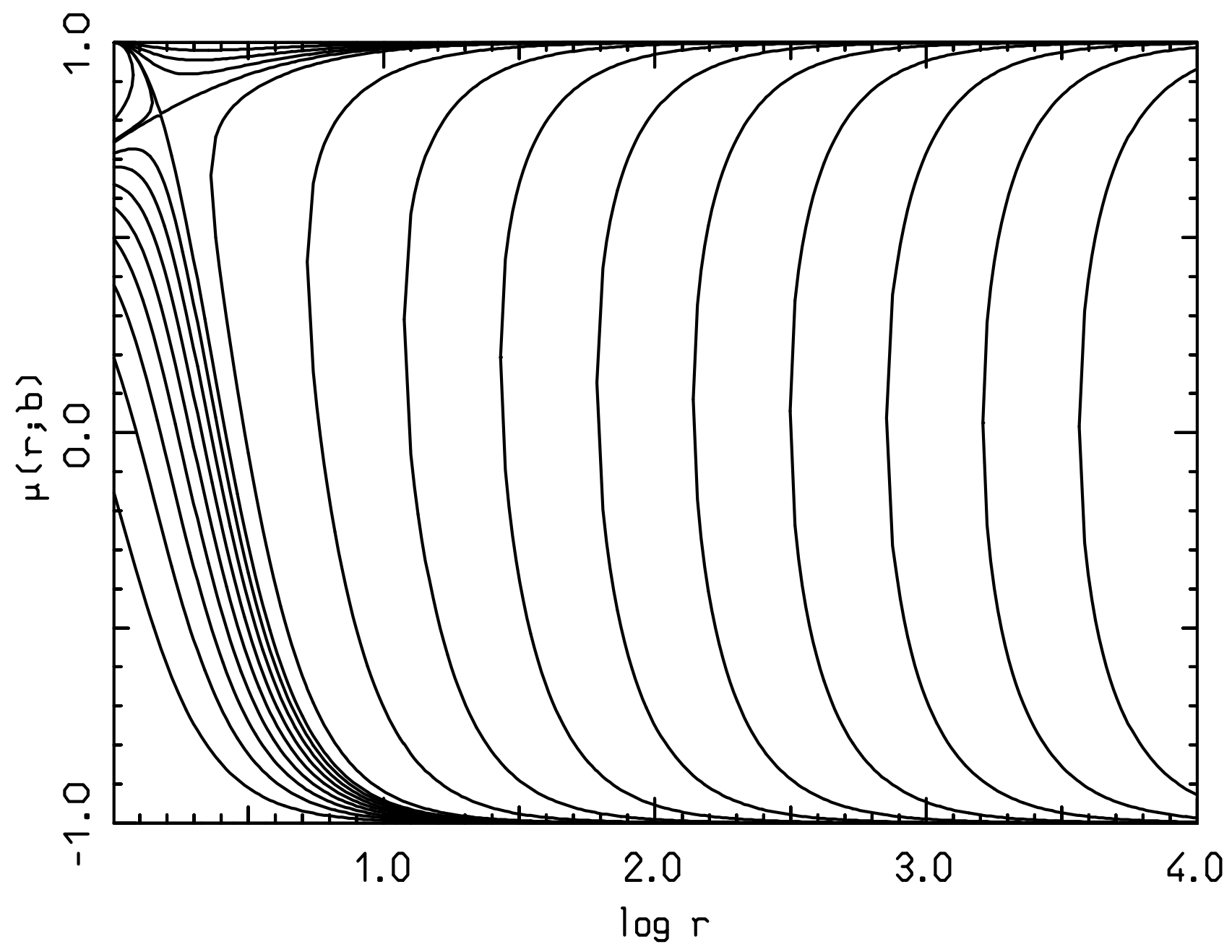




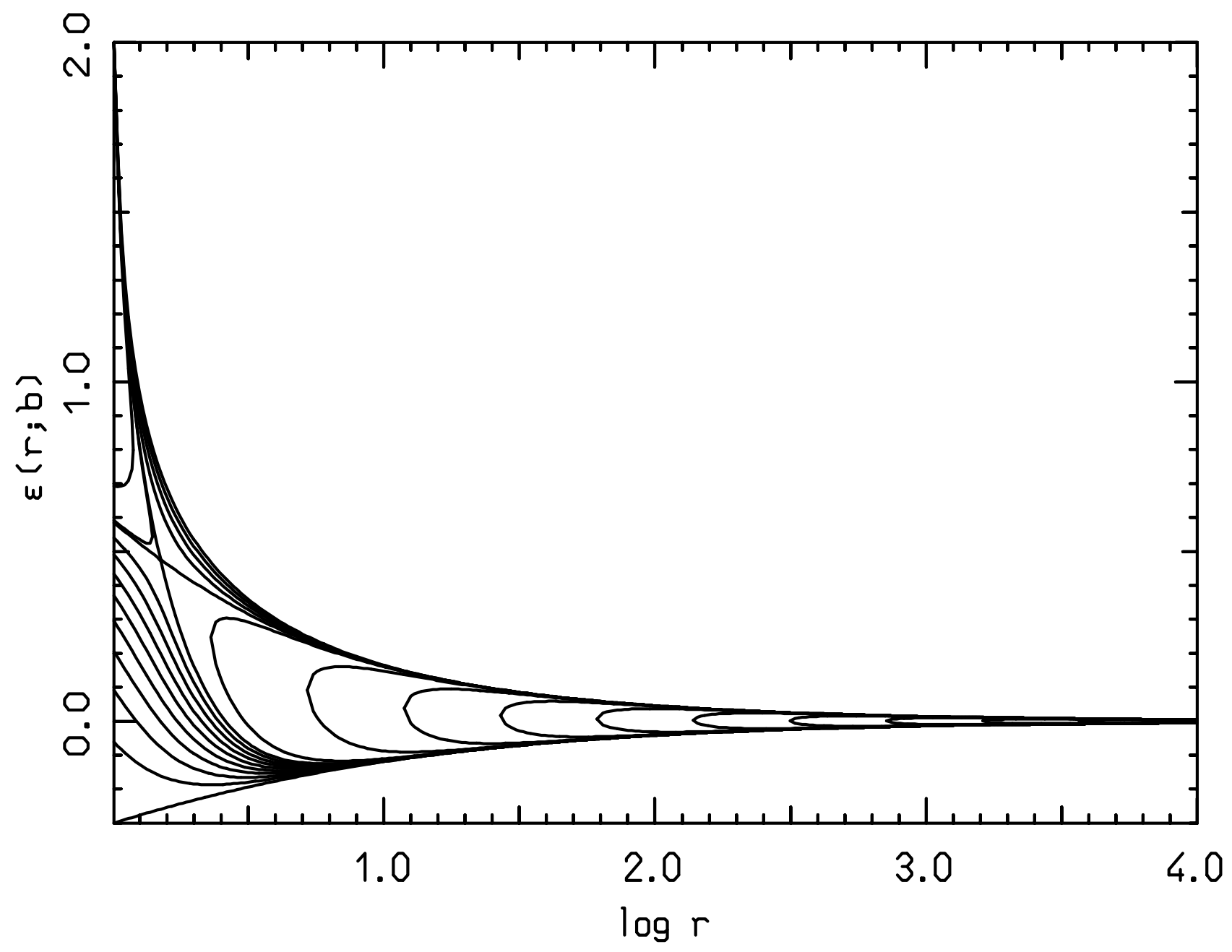




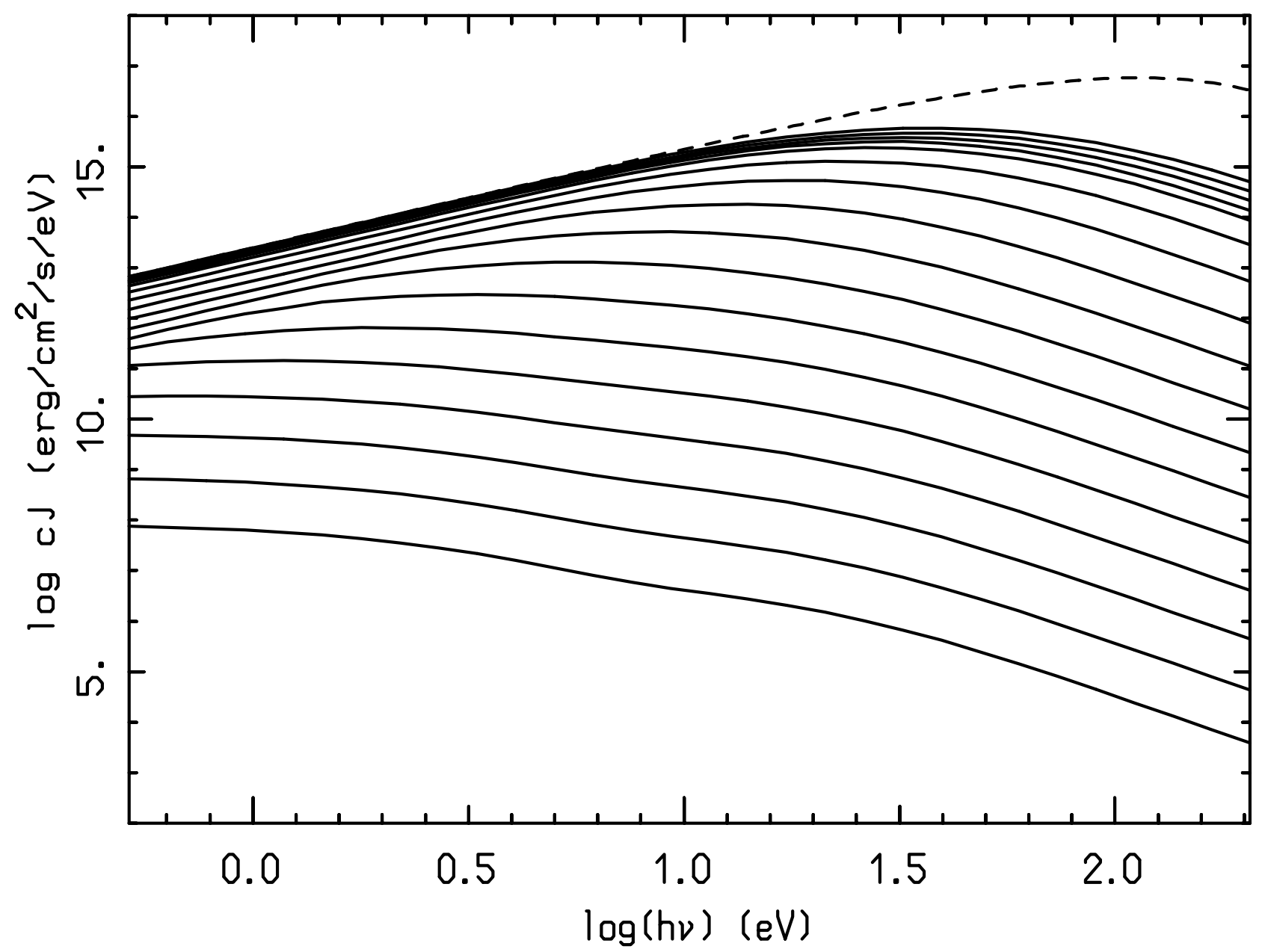




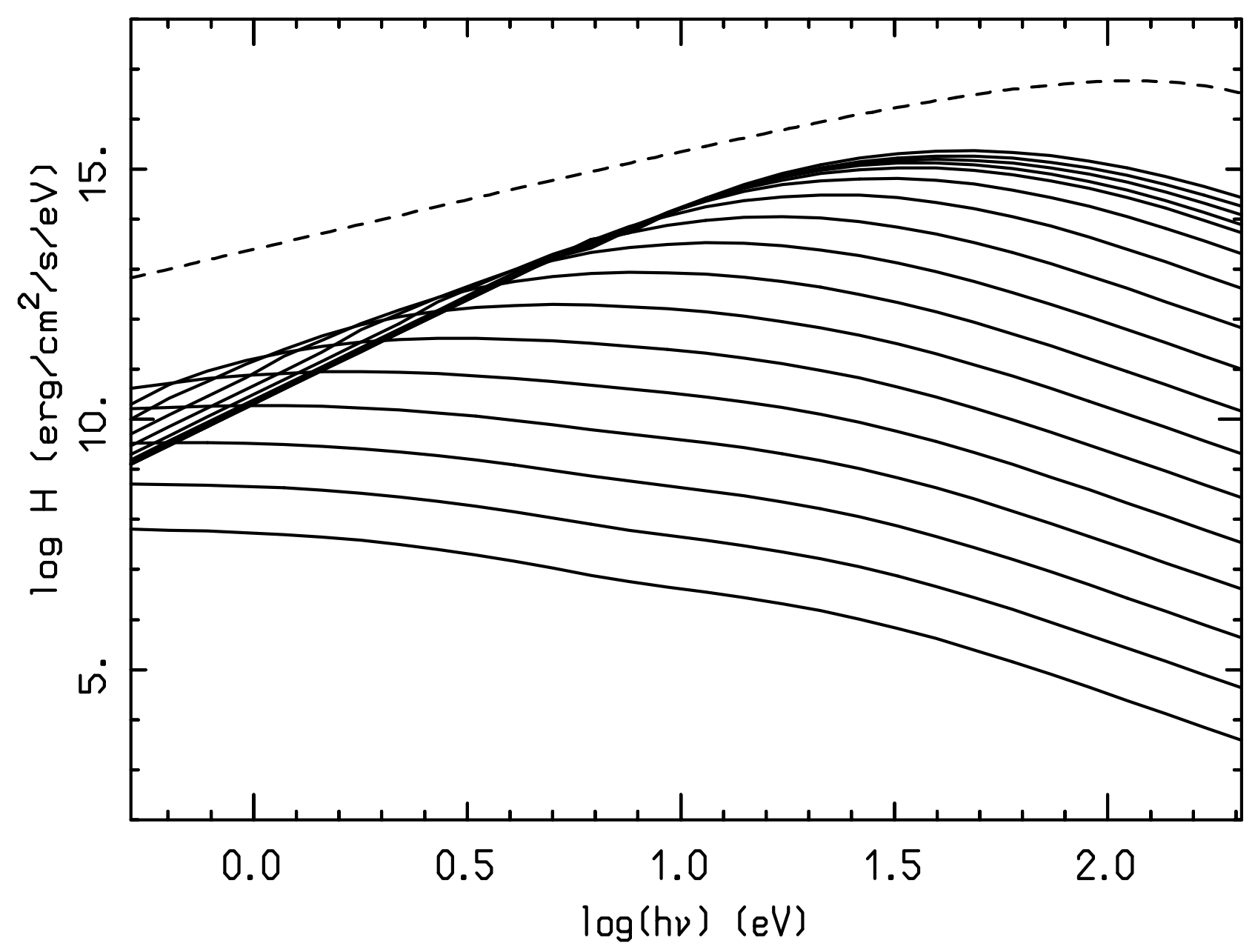




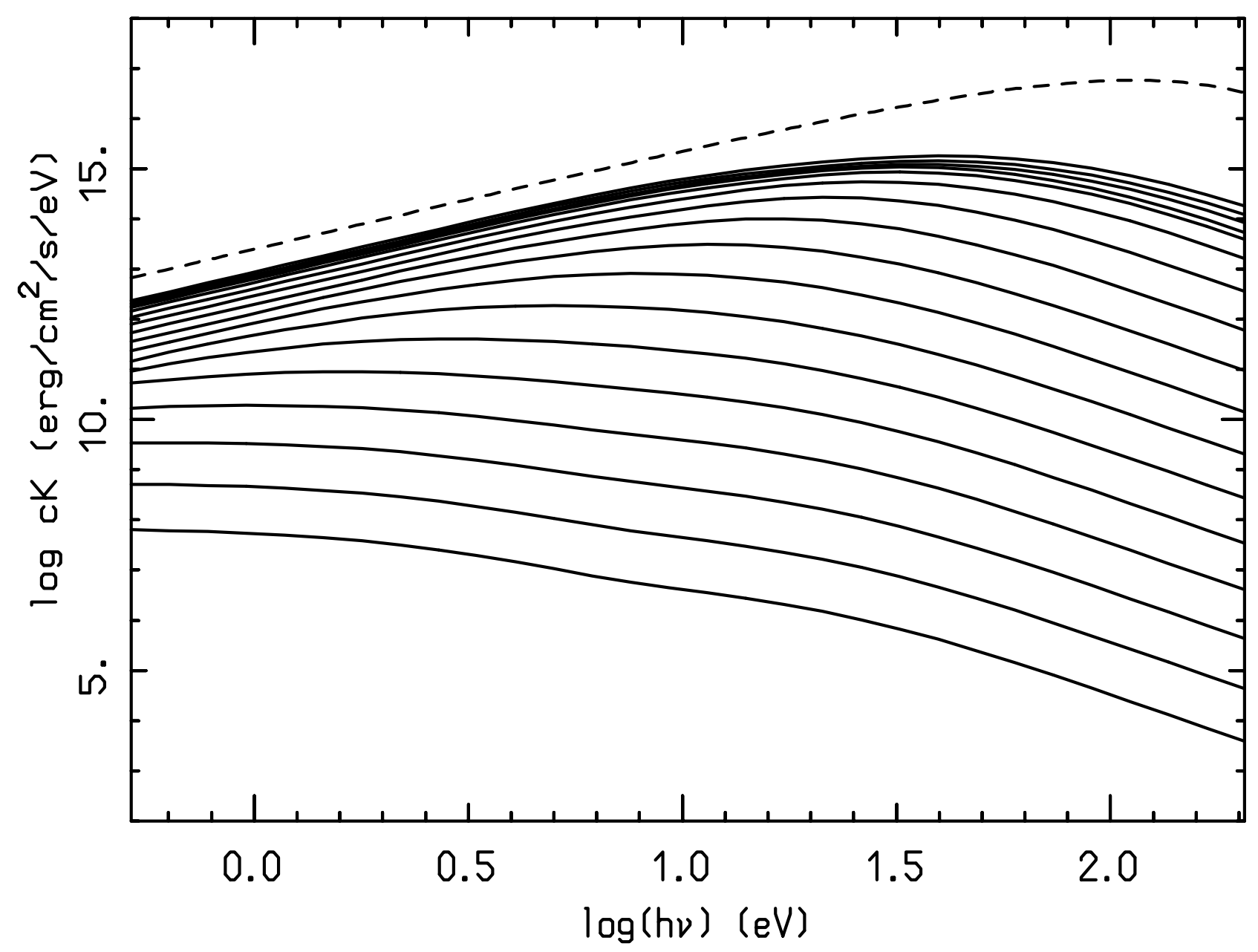




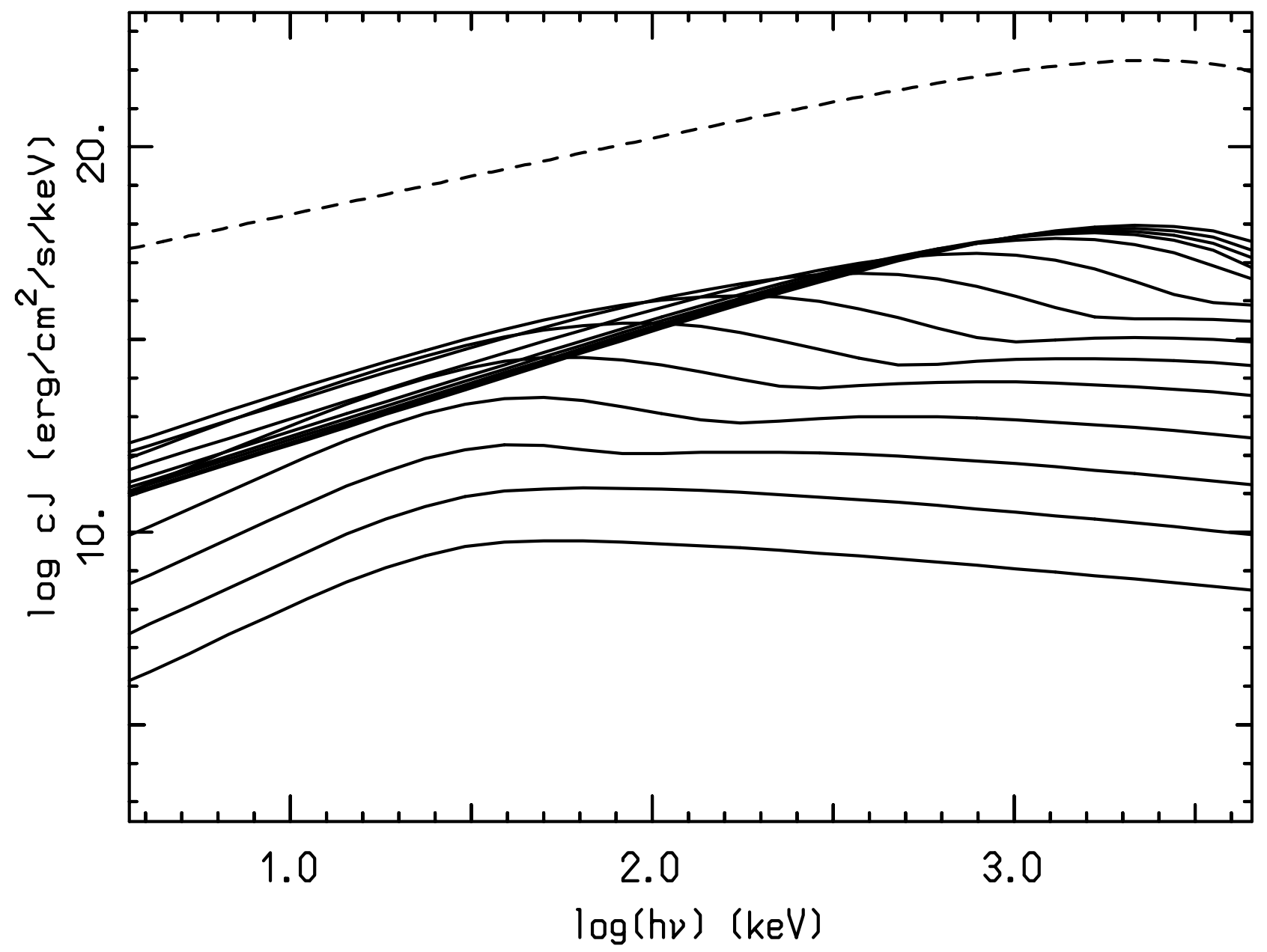




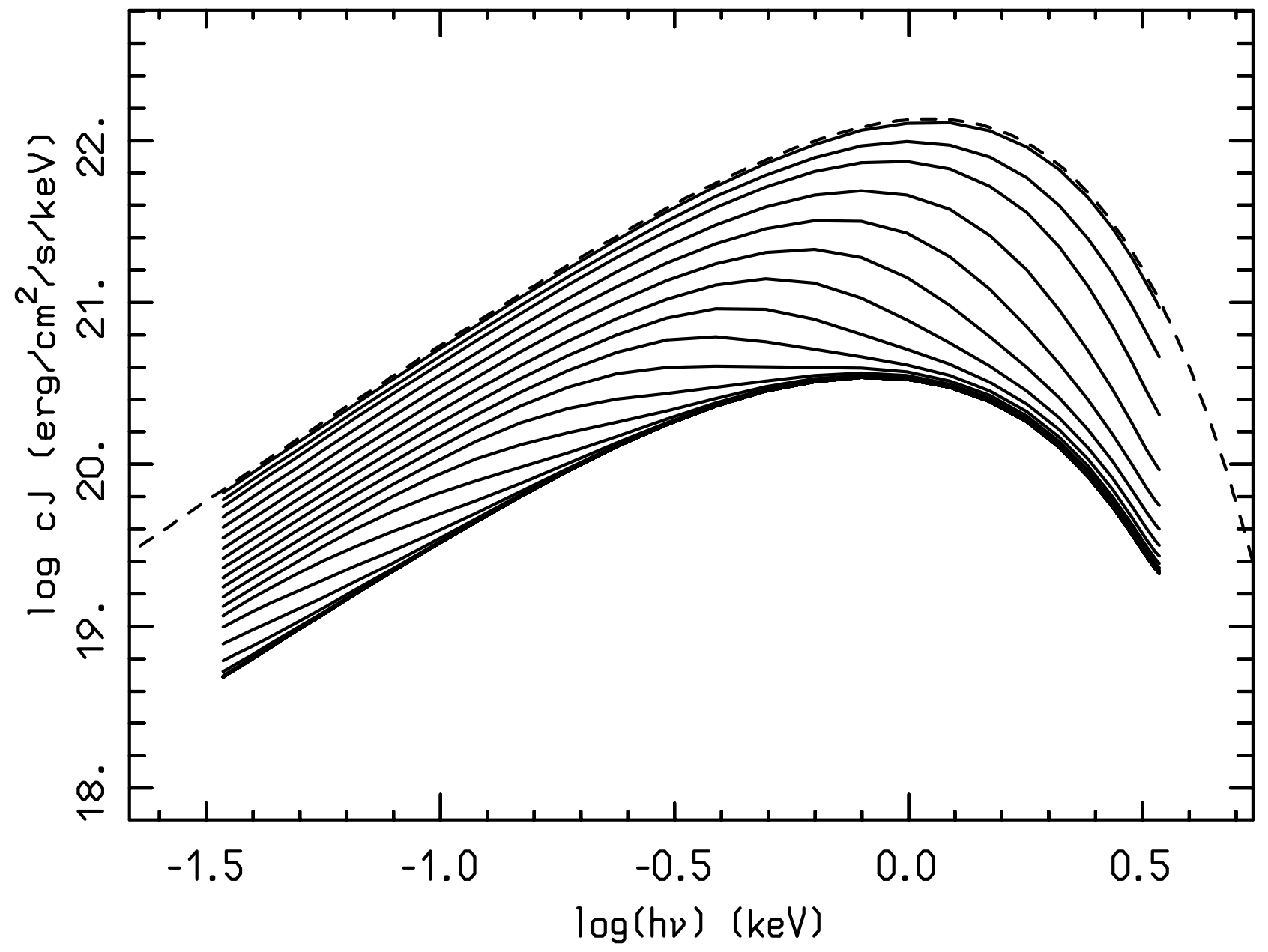




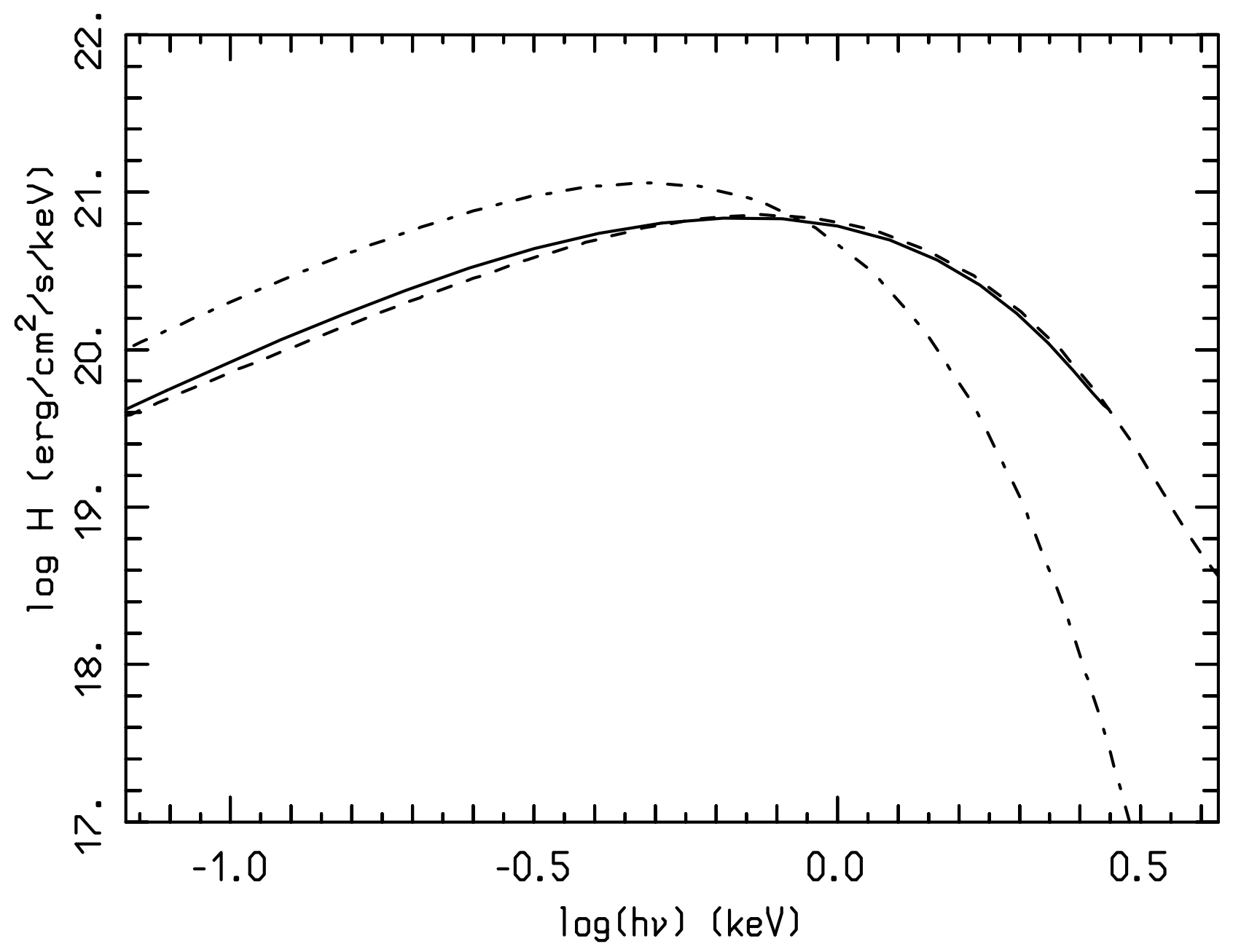




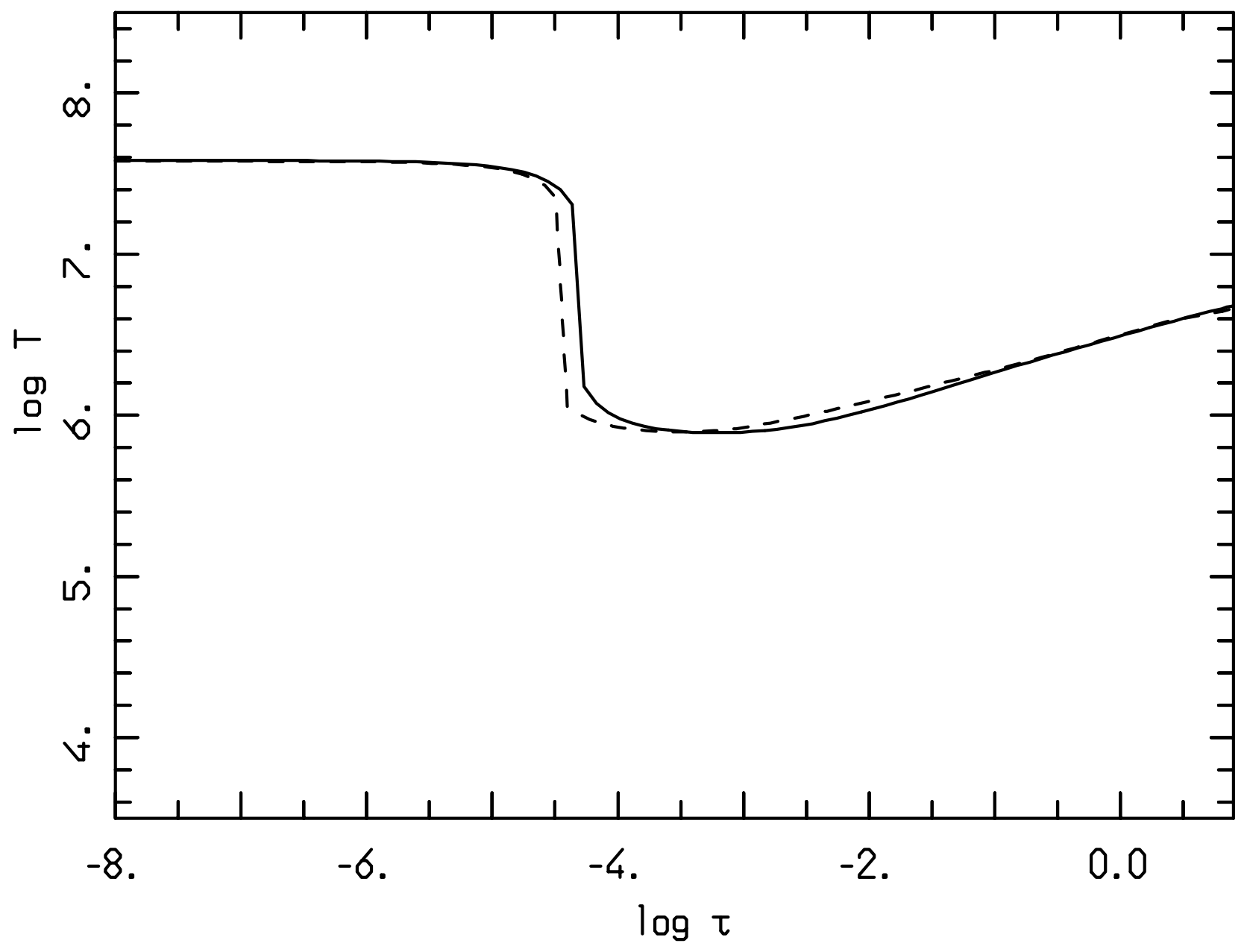

